\title{
The Valuation of Long-Term Exchange Options in the German Electricity Market
}

\author{
Jan Marckhoff*
}

Version: May 4, 2009

\begin{abstract}
This paper analyzes the valuation of monthly Physical Transmission Rights (PTRs) on the German-Dutch interconnector. From a financial perspective, PTRs are exchange options written on the German and Dutch day-ahead electricity price. We extend the famous exchange option model by Margrabe (1978) and include jumps in the underlying prices. We develop a quasi closed-form solution for our exchange option model and compare its pricing performance to the basic model without jumps for all monthly PTRs between 2001 and 2008. Our results show that the inclusion of jumps significantly reduces the Root Mean Squared Error (RMSE) between model and market prices. We further show that the inclusion of de-spiked prices into both models also improves their pricing performance. Overall, the empirical results show that monthly PTR options are clearly undervalued compared to model prices and even strictly cheaper than the corresponding futures contracts.
\end{abstract}

JEL classification: C13; G13; Q40.

Keywords: Electricity; Physical Transmission Right; Margrabe; MCMC.

\footnotetext{
*University of Bamberg, Chair of Financial Controlling, Kaerntenstr. 7, 96052 Bamberg, Germany, jan.marckhoff@uni-bamberg.de, Tel: +49 951863 2093, Fax: +49 9518632092.

We would like to thank EEX AG, APX Group, ENDEX N.V. and Tennet TSO for kindly providing the data used in this study. We also appreciate valuable comments and suggestions by Alexander Forstbach, Matthias Muck and Jens Wimschulte.
} 


\section{Introduction}

In 1996, the liberalization of European electricity markets started with the adoption of the European Commission's Directive 96/92/EG. Ever since, cross-border electricity flows have continuously been increasing in size and therefore in relevance. In 2007, 12.6\% of the total electricity consumption in all UCTE countries was delivered cross-border. ${ }^{1}$ As the international electricity markets exhibit distinct sources of risk, the growing internationalization also increases the demand for an adequate risk management capable of coping with these unique characteristics. ${ }^{2}$

The risks associated with cross-border electricity markets can generally be separated, analogous to other commodity markets, into a physical and a financial component. Financial risk is concerned with unexpected unfavorable price movements. Although financial risk is inherent in basically all financial and commodity markets, it is considerably more important in electricity markets due to the extreme erratic price fluctuations compared to other markets. Physical risk in electricity markets comprises the ability to deliver on time. Although physical risk is also apparent in other commodity markets, it is of special interest for electricity due to its unique characteristics. Here, two main attributes are of particular importance. First of all, electricity is in general not considered to be storable at a reasonable cost. Although electricity can be indirectly stored via pumped storage facilities, these are currently not available on a large scale and therefore only of minor relevance. ${ }^{3}$ Due to the non storability of electricity, shocks in demand and supply cannot be dampened by existing inventory resulting in the typical erratic trajectory of electricity prices. Second, electricity is grid bound, i.e. delivery is only possible via an existing power grid. In addition, electricity immediately spreads across the entire grid, once it is injected into the system. Therefore, a simple point to point delivery in the electricity market is not feasible and even congested cables not initially intended to carry a load could hamper electricity flows. Although this problem is evident in both, national and international electricity markets, it is more severe in cross-border connections as the respective capacities are scarcer. While the limited transportation possibilities of electricity in international electricity markets clearly involve physical risk, congestion and the resulting price difference in neighboring countries

\footnotetext{
${ }^{1}$ The Union for the Co-ordination of Transmission of Electricity (UCTE) is the association of all transmission system operators (TSO) in the continental European area. The UCTE currently comprises 29 TSOs from 24 countries.

${ }^{2}$ Armstrong and Galli (2005) as well as Zachmann (2008) discuss the ongoing process of connecting European electricity markets.

${ }^{3}$ One exception might be Norway where over $90 \%$ of total electricity production is generated by hydropower plants. See Von der Fehr et al. (2005) for a discussion of the relevance of hydropower in the Nordic electricity market.
} 
introduce additional financial risk to market participants.

In order to manage the risk in cross-border electricity markets, Physical Transmission Rights (PTRs) have been introduced in Europe. ${ }^{4}$ PTRs are option contracts that securitize capacity of cross-border power grid interconnections for a specific period of time. Therefore, these contracts allow for the transmission of electricity between two markets. This right inherent in PTRs offers the chance of buying electricity in one region $A$ and selling it in another region $B$. PTRs therefore allow for profitable transactions in the case of diverging regional prices. From a financial perspective, this transaction results in the exchange of two electricity prices. Thus, the payoff of a PTR can be written as

$$
P T R=\max [B-A ; 0]
$$

Based on this result, PTRs can be considered as exchange options. Margrabe (1978) was the first to discuss the valuation of exchange options. He develops a closed-form solution for this type of option in case both assets follow Itô processes. ${ }^{5}$ The Margrabe model has since been applied extensively to the valuation of various contracts. Gay and Manaster (1984) state, for example, that the quality option implicit in wheat futures at the Chicago Board of Trade (CBOT) can be viewed as an exchange option which the authors price using the Margrabe model. Hemler (1990) shows the same for the delivery option of U.S. treasury bond futures at CBOT and Johnson and Tian (2000) value indexed executive stock options based on the Margrabe formula. Albizzati and Geman (1994) extend the Margrabe formula to include stochastic interest rates and use their model for the valuation of the surrender option inherent in life insurance contracts. Besides the discussed implicit exchange options, Fu (1996) applies the Margrabe formula to value explicit options to exchange two interest rates. ${ }^{6}$

In addition to the application of the Margrabe formula, several authors extend the underlying model. Carr (1988) considers sequential exchange options where exercise induces further exchange options and proposes a closed-form solution. Grinblatt and Titman (1989) extend the Margrabe model to the three asset case and Gerber and Shiu (1996) develop a solution for the valuation of perpetual exchange options. In case of

\footnotetext{
${ }^{4}$ Besides PTRs, Financial Transmission Rights (FTRs) as well as Contracts for Difference (CfDs) have been introduced in Europe to hedge solely financial risk. See Kristiansen (2004) for an overview of various risk management products in cross-border electricity markets and Marckhoff and Wimschulte (2009) for a detailed discussion of CfDs.

${ }^{5}$ Note that at the same time as Margrabe, Fischer (1978) derives an identical formula for the valuation of exchange options. Throughout this paper, however, we refer to the exchange option formula as the Margrabe model.

${ }^{6}$ See Gastineau (1993) and Margrabe (1993) for further applications of the Margrabe exchange option model.
} 
non-normal distributions of the underlying assets Cherubini and Luciano (2002) use copula functions for the valuation of exchange options. Another approach follows Li (2008). He uses a multivariate Gram-Chalier approximation in order to derive the value of an exchange option in case of non-normal asset distributions.

In this paper, we propose an extension to the Margrabe formula by including jumps in both underlying asset prices. Following Cartea and Figueroa (2005), we propose a mean-reverting jump-diffusion model and derive a quasi closed-form solution for the valuation of exchange options based on Merton (1976). We use our model for the valuation of monthly PTR options on the German and Dutch interconnector, i.e. options on the spread between the German and Dutch electricity price. We analyze the pricing performance of our model compared to the standard case without jumps. As electricity prices are highly erratic, including jumps in order to match distributional and trajectorial characteristics of electricity prices significantly improves the model performance. ${ }^{7}$ However, since jumps in electricity prices generally fade within a few days, their impact on the valuation of monthly electricity options is not unambiguous. ${ }^{8}$ In addition to the incorporation of jumps in the exchange option model, we further test the effect of jumps in the underlying electricity prices when those prices serve as input factors to the valuation model. Due to the high mean-reversion speed in electricity markets, prices exhibit only a loose intertemporal connection. Thus, today's electricity prices are only a vague indicator of future prices and the inclusion of observed electricity prices could distort resulting option values. This effect is particularly prevailing in the case of jumps in the underlying prices. We therefore analyze the pricing performance of both models, i.e. diffusion and jump-diffusion model, using only de-spiked electricity prices.

Our dataset includes all 96 monthly PTR options between 2001 and 2008 in addition to daily baseload prices for Germany and the Netherlands. Daily prices are available for all 2,922 days between January 1, 2001 and December 31, 2008 and are mainly used for the estimation of our model parameters and latent state variables. For estimating the empirical (or physical) parameters, we refer to the method of Markov Chain Monte Carlo (MCMC). This method of estimation is widely used for equity models and, among others, applied by Eraker et al. (2003) as well as Eraker (2004) and more recently by Rodrigues and Schlag (2009).

This paper contributes to various aspects of recent research. First, we derive a quasi closed-form solution for the valuation of exchange options in the case of jumps in the

\footnotetext{
${ }^{7}$ See Bierbrauer et al. (2007) for a comparison of various models for German electricity prices.

${ }^{8}$ Dempster et al. (2008), for example, propose a two factor diffusion model for the valuation of long term spread options in commodity markets.
} 
underlying assets. Second, we empirically investigate the pricing performance of the extended Margrabe model for monthly PTR contracts. Third, we analyze the impact of de-spiked prices on the valuation of both exchange option models, i.e. diffusion and jump-diffusion. Finally, we offer a detailed analysis of the pricing of monthly PTR options on the German and Dutch interconnector. Our findings show that the inclusion of jumps significantly improves the pricing performance of the exchange option model. In addition, the usage of de-spiked prices leads to a further improvement of the pricing performance. Although the first effect is more pronounced, the latter is still significant. Overall, our empirical results show that monthly PTR options are generally underpriced. This confirms the prior findings of Dieckmann et al. (2008).

In the course of our analysis, we start with a short discussion of the national electricity markets of Germany and the Netherlands as well as their cross-border interconnection. ${ }^{9}$ Afterwards, we introduce our exchange option model and derive the closed-form solution for the diffusion model and a quasi closed-form solution for the jump-diffusion model respectively. We then describe the estimation of our empirical and risk-neutral parameters and also shortly explain the process of de-spiking the underlying electricity prices. The following section presents and discusses the results of our analysis before the last section concludes.

\section{German-Dutch Electricity Market}

\subsection{National Electricity Markets}

In Germany and the Netherlands, the exchange based electricity markets are separated into a spot and a derivatives market. In Germany, both are organized by the European Energy Exchange (EEX). ${ }^{10}$ The spot market is basically a day-ahead market, where electricity is auctioned on each working day for every hour of the next day or days in case of a holiday or a weekend. The derivatives market at the EEX consists of options and futures contracts, whereof the latter are by far more liquidly traded. Futures contracts are further divided into physically and financially settled contracts. The derivatives market is significantly larger compared to the spot market. While the trading volume of the spot market in 2007 was about 124 TWh, the derivatives market had a volume 1.150 TWh. The Dutch electricity market is comparable concerning its exchange based

\footnotetext{
${ }^{9}$ See Marckhoff and Muck (2009) for a more detailed discussion of the German and Dutch national electricity markets as well as their respective cross-border market.

${ }^{10}$ In January 2008, the French electricity exchange Powernext and the EEX founded the EEX Power Derivatives $\mathrm{GmbH}$ intended to constitute a joint platform for derivatives trading in both countries. While the EEX owns an $80 \%$ stake in the new compony, Powernext holds the remaining $20 \%$.
} 
trading. The market also distinguishes between spot and derivatives trading. The spot market is organized by the Amsterdam Power Exchange (APX) and also mainly consists of a day-ahead market. ${ }^{11}$ The set up of the derivatives market is basically identical to the German one, however, it is organized separately by the European Energy Derivatives Exchange (ENDEX). The spot market volume at APX in 2007 was about 21 TWh compared to a derivatives market volume of $101 \mathrm{TWh}$.

Despite similarities in the exchange based market set up, the German and Dutch electricity markets differ significantly concerning their physical electricity markets. While in the Netherlands, as one of the largest natural gas producers in the world, gas power plants contribute considerably to the power generation mix, other fuels only play a minor role in electricity production. ${ }^{12}$ Germany in contrast, exhibits a more diversified mix concerning its power generation. Although coal (both hard and lignite) as well as nuclear power plants constitute the major share in German electricity production, natural gas and renewables also contribute significantly to the power generation mix. Therefore, the Dutch electricity market exhibits a more convex supply curve resulting in generally higher and more erratic electricity prices. Figure 1 shows the logarithm of daily baseload prices, i.e. the arithmetic mean of the 24 hourly prices, for Germany and the Netherlands between 2001 and 2008. We refer to the logarithm of prices as these constitute the basis for our model.

\section{[Insert Figure 1 here]}

Daily spot prices show a characteristic pattern. First of all, both trajectories are extremely erratic whereof Dutch spot prices are significantly more erratic than German ones. This applies for the regular variations as well as the occasional jumps. Second, electricity prices are mean-reverting, i.e. they rapidly tend back to a specific mean-reversion level. This level, however, is not constant over time but rather exhibits a seasonal trend. The trend is evident on a weekly as well as an annual basis due to seasonal patterns in demand. In addition to the characteristics of national electricity prices, the co-movement of both prices is also apparent. The more erratic prices in the Netherlands are explained by the specific power generation mix and the co-movement is a clear sign of connecting European electricity markets. Table 1 provides detailed descriptive statistics for the logarithm of daily baseload prices in Germany and the Netherlands. Since we focus on the valuation of monthly PTRs, we further show the monthly average of both electricity prices.

\footnotetext{
${ }^{11}$ Since 2006, there exists an intraday market at EEX and APX, where electricity is traded continuously for certain time blocks.

${ }^{12}$ In 2007, the Netherlands produced about 76.3 billion cubic meters of natural gas which corresponds to almost $39 \%$ of the European Union's natural gas production. See http://www.cia.gov for details.
} 


\section{[Insert Table 1 here]}

Section A of Table 1 confirms the observed characteristics from Figure 1. In both countries electricity prices exhibit large variations confirmed by the high variance as well the kurtosis. Further, prices in the Netherlands are generally above German prices and in both countries positive jumps are more severe and observed more frequently than negative ones as indicated by the skewness. The statistics of daily prices clearly indicate that the distribution is far from normality. This indication is confirmed by Jarque-Bera values of 692 for Germany and 431 for the Netherlands respectively. Section B shows that the monthly average of daily spot prices is not as wide spread, i.e. the minimum increases and the maximum decreases. While the variance is also reduced, the skewness increases and the kurtosis decreases even further. Although the Jarque-Bera values are dramatically reduced to 60 and 64 for Germany and the Netherlands due to the smaller sample size, prices still depart considerably from being normally distributed. ${ }^{13}$ Thus, using monthly average values does not significantly shift the distributional characteristics of prices towards the normal distribution.

\subsection{The German-Dutch Cross-Border Market}

The German and Dutch electricity markets are currently connected via three highvoltage $(380 \mathrm{kV})$ power lines. The capacity of these interconnectors is managed solely via PTR options. Three different contract types are distinguished which differ only in terms of the length of their delivery period. These contract types comprise hourly, monthly and yearly PTR options where every contract has a volume of 1MW. All PTRs are auctioned explicitly by Auction BV, a $100 \%$ subsidiary of the Dutch transmission system operator (TSO) TenneT. ${ }^{14}$ Hourly PTRs are auctioned for every hour of the following day and cover only a single hour. Monthly PTRs are auctioned on the 10th working day of the month preceding the delivery month and constitute a portfolio of hourly PTRs for each hour of the delivery month. Yearly PTRs are auctioned on the first working day following the 27th of September in the year preceding the delivery year. In case of remaining capacity, there is a second auction for yearly PTRs on the first working day after the 27th of November. Analogous to monthly contracts, yearly PTRs are portfolios of hourly PTRs for each hour of the delivery year. Generally, one third of the available capacity is reserved for each contract type. The price of the auction is zero

\footnotetext{
${ }^{13}$ The Jarque-Bera test statistics are asympotically $\chi^{2}$ distributed with $2 \mathrm{df}$. The $99 \%$ quantile of this distribution is 9.21 and therefore significantly below the estimated values.

${ }^{14}$ PTRs are auctioned seperately for each direction. Further, there are different PTRs available for the two German TSOs RWE TSO and E.ON Netz. Throughout this paper, all analyses refer to RWE TSO interconnections from Germany to the Netherlands as these contracts are by far more liquid.
} 
in case the available capacity exceeds demand and is determined by the lowest successful bid otherwise. If several lowest bids exist, the capacity is allocated proportional to the requested volume. Table 2 shows the descriptive statistics of all monthly PTR options between January 2001 and December 2008.

\section{[Insert Table 2 here]}

\section{Model}

\subsection{Diffusion Processes}

Our diffusion model is based on the one factor model of Lucia and Schwartz (2002). We model the logarithm of the spot price, i.e. $\ln P_{t}$, which can be decomposed into a deterministic trend $f_{P}(t)$ and a stochastic component $S_{t}$. As we use identical models for the German and Dutch electricity prices, we refrain from stating our model separately for each country. We rather use a general notation where $P_{t} \in\left\{G_{t}, D_{t}\right\}$ refers to the German $\left(G_{t}\right)$ as well as the Dutch $\left(D_{t}\right)$ spot price at time $t$. Further, the stochastic component $S_{t}$ represents both, the German $\left(X_{t}\right)$ and Dutch $\left(Y_{t}\right)$ stochastic component, i.e. $S_{t} \in\left\{X_{t}, Y_{t}\right\}$. This notation is also used when subscribing the deterministic functions as well as the parameters of our model. Therefore, we can write for the logarithm of the spot price

$$
\ln P_{t}=f_{P}(t)+S_{t}
$$

The deterministic function is used to filter out the annual and intra-week seasonality inherent in German and Dutch electricity prices. $S_{t}$ therefore only contains the stochastic fluctuations around the mean-reversion level of zero. In order to model the mild fluctuation of electricity prices in the short run and the reversion to a seasonal trend in the long run, $S_{t}$ follows an Ornstein-Uhlenbeck process. Hence, $S_{t}$ is governed by the following SDE under the empirical (or physical) measure $\mathcal{P}$

$$
d S_{t}=-\kappa_{P} S_{t} d t+\sigma_{P} d W_{P}(t)
$$

$\kappa_{P}$ and $\sigma_{P}$ are constant parameters, where the former is the mean-reversion speed while the latter represents the volatility of $S_{t}$. The increments of the Wiener processes, i.e. $d W_{G}(t)$ and $d W_{D}(t)$, are assumed to be correlated. In order to model the correlation $\rho$ between German and Dutch electricity prices, we assume that the increments of the Wiener process in Dutch electricity prices can be written as $d W_{D}=$

$\rho d W_{G}(t)+\sqrt{1-\rho^{2}} d Z_{D}(t)$. Here, $d Z_{D}(t)$ is another increment of a Wiener process independent of $d W_{G}(t)$. Therefore, the relation between the Wiener processes can be 
described as

$$
\begin{aligned}
E\left[d W_{G}(t) d Z_{D}(t)\right] & =0 \\
E\left[d W_{G}(t) d W_{D}(t)\right] & =\rho d t .
\end{aligned}
$$

In order to derive the SDE of the spot price $P_{t}$, we apply Itô's Lemma and obtain

$$
d P_{t}=\kappa_{P}\left[\gamma_{P}(t)-\ln P_{t}\right] P_{t} d t+\sigma_{P} P_{t} d W_{P}(t)
$$

where

$$
\gamma_{P}(t)=\frac{1}{\kappa_{P}}\left(\frac{1}{2} \sigma_{P}^{2}+\frac{d f_{P}(t)}{d t}\right)+f_{P}(t)
$$

When deriving the risk-neutral process for $S_{t}$ we use the Girsanov theorem, see Girsanov (1960), to change to the risk-neutral measure $\mathcal{Q}$. The risk-neutral SDE for $S_{t}$ then follows $\operatorname{as}^{15}$

$$
d S_{t}=\kappa_{P}\left(-\frac{\sigma_{P}}{\kappa_{P}} \theta_{P}-S_{t}\right) d t+\sigma_{P} d W_{P}^{Q}(t)
$$

$\theta_{P}$ indicates the constant market price of diffusion risk which is market specific and therefore differs for Germany and the Netherlands. The change to the risk-neutral measure leads to an altered mean-reversion level, while the mean-reversion speed as well as the volatility remain constant. The measure change results, after applying Itô's Lemma, in the following SDE for the spot price $P_{t}$

$$
d P_{t}=\kappa_{P}\left[\gamma_{P}^{Q}(t)-\ln P_{t}\right] P_{t} d t+\sigma_{P} P_{t} d W_{P}^{Q}(t)
$$

where

$$
\gamma_{P}^{Q}(t)=\frac{1}{\kappa_{P}}\left(\frac{1}{2} \sigma_{P}^{2}+\frac{d f_{P}(t)}{d t}\right)+f_{P}(t)-\frac{\sigma_{P}}{\kappa_{P}} \theta_{P}
$$

Since we model the logarithm of the spot price, we derive the risk-neutral process for $\ln P_{t}$, which is the sum of the deterministic trend and the solution to the above stated risk-neutral SDE for $S_{t}{ }^{16}$ The process for the logarithm of the spot price follows as

$$
\ln P_{t}=f_{P}(t)+\left(\ln P_{0}-f_{P}(0)\right) e^{-\kappa_{P} t}-\frac{\sigma_{P}}{\kappa_{P}} \theta_{P}\left(1-e^{-\kappa_{P} t}\right)+\sigma_{P} \int_{0}^{t} e^{\kappa_{P}(s-t)} d W_{P}^{Q}(s) .
$$

Thus, the logarithm of the spot price is normally distributed with expectation and

\footnotetext{
${ }^{15}$ We use the super index $\mathcal{Q}$ to indicate the risk-neutral measure. For the empirical measure $\mathcal{P}$, we omit the super index.

${ }^{16}$ See Mikosch (1999) for a detailed discussion of the solution to the SDE.
} 
variance of

$$
\begin{aligned}
E^{Q}\left[\ln P_{t}\right] & =f_{P}(t)+\left(\ln P_{0}-f_{P}(0)\right) e^{-\kappa_{P} t}-\frac{\sigma_{P}}{\kappa_{P}} \theta_{P}\left(1-e^{-\kappa_{P} t}\right), \\
\operatorname{Var}^{Q}\left[\ln P_{t}\right] & =\frac{\sigma_{P}^{2}}{2 \kappa_{P}}\left(1-e^{-2 \kappa_{P} t}\right) .
\end{aligned}
$$

The risk-neutral expectation of the spot price is ${ }^{17}$

$$
\begin{aligned}
E^{Q}\left[P_{t}\right]= & \exp \left(f_{P}(t)+\left(\ln P_{0}-f_{P}(0)\right) e^{-\kappa_{P} t}\right) \\
& \cdot \exp \left(\frac{\sigma_{P}^{2}}{4 \kappa_{P}}\left(1-e^{-2 \kappa_{P} t}\right)-\frac{\sigma_{P}}{\kappa_{P}} \theta_{P}\left(1-e^{-\kappa_{P} t}\right)\right) .
\end{aligned}
$$

\subsection{Option Price without Jumps}

In order to determine the value of an option to exchange the German and Dutch electricity price, we first derive the PDE for the option price. Our approach exploits the fact that the option price is linear homogenous in both electricity prices. ${ }^{18}$ Therefore, we can use Euler's theorem to write the value of the option at time $t$, i.e. $C_{t}$, as

$$
C_{t}-\frac{\partial C_{t}}{\partial G_{t}} G_{t}-\frac{\partial C_{t}}{\partial D_{t}} D_{t}=0
$$

After differentiating the equation for $d C_{t}$ reads as

$$
d C_{t}-\frac{\partial C_{t}}{\partial G_{t}} d G_{t}-\frac{\partial C_{t}}{\partial D_{t}} d D_{t}=0
$$

We finally attain the PDE for the option price by applying Itô's Lemma to $d C_{t}$ and substituting for $d C_{t}$ according to the above equation. This establishes the following PDE

$$
\frac{\partial C_{t}}{\partial t_{t}}+\frac{1}{2}\left[\frac{\partial^{2} C_{t}}{\partial G_{t}^{2}} G_{t}^{2} \sigma_{G}^{2}+\frac{\partial^{2} C_{t}}{\partial D_{t}^{2}} D_{t}^{2} \sigma_{D}^{2}+2 \frac{\partial C_{t}}{\partial G_{t} \partial D_{t}} G_{t} D_{t} \sigma_{G} \sigma_{D} \rho\right]=0 .
$$

This PDE is identical to the PDE derived by Margrabe (1978). Therefore, our solution to the PDE is also identical since we apply the same boundary and terminal value assumptions. The value of the option at time $t$ to exchange the German and Dutch

${ }^{17}$ Given a $\log$-normally distributed variable $\varepsilon$, i.e. $\ln \varepsilon \sim N\left(\mu, \sigma^{2}\right)$, the expectation of $\varepsilon$ can be derived as $E\left[e^{\ln \varepsilon}\right]=e^{\mu+\frac{1}{2} \sigma^{2}}$. The risk-neutral expectation of $P_{t}$ is identical to the forward price with maturity in $t$.

${ }^{18}$ The option value at maturity is $C_{T}=\left(D_{T}-G_{T}\right)^{+}$which is linear homogenous in both electricity prices, i.e. for any constant $\alpha \in \mathbb{R}_{0}^{+}$we have $\alpha C_{T}=\left(\alpha D_{T}-\alpha G_{T}\right)^{+}$. The option value at time $t$, with $t<T$, is just the discounted value of $C_{T}$ and therefore also linear homogenous in both electricity prices. 
electricity price with time to maturity $\tau=T-t$, follows as ${ }^{19}$

$$
C_{t}\left(G_{t}, D_{t}\right)=D_{t} \Phi\left(d_{1}\right)-G_{t} \Phi\left(d_{2}\right)
$$

where $\Phi(\cdot)$ denotes the cumulative normal distribution function and

$$
\begin{aligned}
d_{1} & =\frac{\ln \left(\frac{D_{t}}{G_{t}}\right)+\frac{1}{2} \sigma^{2} \tau}{\sigma \sqrt{\tau}}, \\
d_{2} & =d_{1}-\sigma \sqrt{\tau}, \\
\sigma^{2} & =\sigma_{G}^{2}+\sigma_{D}^{2}-2 \sigma_{G} \sigma_{D} \rho .
\end{aligned}
$$

Our solution shows that the resulting option price is independent of the drift component and thus, the mean-reversion characteristics of the underlying electricity prices. Further, the market price of diffusion risk $\theta_{P}$ has also no influence on the resulting option price.

\subsection{Jump-Diffusion Processes}

Following Cartea and Figueroa (2005), we augment the diffusion model and include lognormal jumps in our underlying, or more precise in the SDE of the stochastic component. The reason for adding jumps when modeling electricity spot prices is obviously justified by the observed prices shown in Figure 1. A pure diffusion model is not capable of mirroring the depicted trajectory. Applying the same notation as above, the SDEs for $S_{t}$ and $P_{t}$ read as

$$
\begin{aligned}
& d S_{t}=-\kappa_{P} S_{t} d t+\sigma_{P} d W_{P}(t)+\ln J_{P, t} d q_{P, t} \\
& d P_{t}=\kappa_{P}\left(\gamma_{P}(t)-\ln P_{t}\right) P_{t} d t+\sigma_{P} P_{t} d W_{P}(t)+P_{t}\left(J_{P, t}-1\right) d q_{P, t},
\end{aligned}
$$

where

$$
\gamma_{P}(t)=\frac{1}{\kappa_{P}} \frac{d f_{P}(t)}{d t}+f_{P}(t) .
$$

$J_{P, t}$ is log-normally distributed, i.e. $\ln J_{P, t} \sim N\left(\mu_{J}, \sigma_{J}^{2}\right)$ and $q_{P, t}$ is a Poisson process independent of $d W_{P}(t)$. In order to derive the SDE for the logarithm of the spot price, we use Itô's Lemma for jump-diffusion processes according to Cont and Tankov (2003) to calculate $d \ln P_{t}$. We receive

$$
d \ln P_{t}=d f_{P}(t)+\kappa_{P}\left(\gamma(t)-\ln P_{t}\right) d t-\frac{1}{2} \sigma_{P}^{2} d t+\sigma_{P} d W_{P}(t)+\ln J_{P, t}
$$

\footnotetext{
${ }^{19}$ The option price formula corresponds to the case where the holder of the PTR receives the Dutch electricity price in exchange for the German one. In order to obtain the PTR option price for the opposite direction, the German and Dutch electricity prices simply need to be exchanged.
} 
Solving this SDE, we attain the following process for $\ln P_{t}$

$$
\ln P_{t}=\frac{\ln P_{0}+\Delta f_{P}(t)}{\left(1+t \kappa_{P}\right)}+\frac{\left(\kappa_{P} \gamma(t)-\frac{1}{2} \sigma_{P}^{2}\right) t}{\left(1+t \kappa_{P}\right)}+\frac{\sigma_{P}}{\left(1+t \kappa_{P}\right)} W_{P}(t)+\frac{1}{\left(1+t \kappa_{P}\right)} \sum_{i=1}^{N_{t}} \ln J_{P, i}
$$

where $\Delta f_{P}(t)=f_{G}(t)-f_{G}(0)$ and $N_{t}$ indicates the number of jumps in $\ln P_{t}$ until time $t$. Since we need the risk-neutral process in order to value the option, we use the Girsanov theorem as applied before. ${ }^{20}$ While the measure change for the diffusion part has the same effect as discussed above, for the jump component, the jump intensity as well as the mean jump size are altered under the risk-neutral measure. In turn, the variance of the jump size remains unchanged, i.e. $\sigma_{J}^{Q}=\sigma_{J}$. Assuming a Poisson distributed jump process and normally distributed jump sizes, the risk-neutral jump intensity $\lambda^{Q}$ and mean jump size $\mu_{J}^{Q}$ are

$$
\begin{aligned}
\lambda^{Q} & =\lambda e^{\mu_{J} \widetilde{\theta}_{P}+0.5 \tilde{\theta}_{P}^{2} \sigma_{J}^{2}}, \\
\mu_{J}^{Q} & =\mu_{J}+\widetilde{\theta}_{P} \sigma_{J}^{2} .
\end{aligned}
$$

$\widetilde{\theta}_{P}$ is the market price of jump risk. Using the aforementioned transform we can write the risk-neutral SDEs as

$$
\begin{aligned}
& d S_{t}=\kappa_{P}\left(f_{P}(t)-\ln P_{t}\right) d t+\sigma_{P} d W_{P}^{Q}(t)+\ln J_{P, t}^{Q} d q_{P, t}^{Q}, \\
& d P_{t}=\kappa_{P}\left(\gamma_{P}^{Q}(t)-\ln P_{t}\right) P_{t} d t+\sigma_{P} P_{t} d W_{P}^{Q}(t)+P_{t}\left(J_{P, t}^{Q}-1\right) d q_{P, t}^{Q},
\end{aligned}
$$

where

$$
\gamma_{P}^{Q}(t)=\frac{1}{\kappa_{P}} \frac{d f_{P}(t)}{d t}+f_{P}(t)-\frac{\sigma_{P}}{\kappa_{P}} \theta_{P}
$$

Under the risk-neutral measure $\mathcal{Q}, q^{Q}$ is still a Poisson process but with intensity $\lambda^{Q}$ and $\ln J^{Q} \sim \mathcal{N}\left(\mu_{J}^{Q}, \sigma_{J}^{2}\right)$. We see that the SDEs for $S_{t}$ as well as $P_{t}$ are almost identical across measures. The only differences are the new mean-reversion level, the altered mean jump size and jump intensity. The SDE for $\ln P_{t}$ follows analogously to the empirical case and we therefore refrain from stating it again. Under the risk-neutral measure, the process for $\ln P_{t}$ is

$\ln P_{t}=\frac{\ln P_{0}+\Delta f_{P}(t)}{\left(1+t \kappa_{P}\right)}+\frac{\left(\kappa_{P} \gamma^{Q}(t)-\frac{1}{2} \sigma_{P}^{2}\right) t}{\left(1+t \kappa_{P}\right)}+\frac{\sigma_{P}}{\left(1+t \kappa_{P}\right)} W_{P}^{Q}(t)+\frac{1}{\left(1+t \kappa_{P}\right)} \sum_{i=1}^{N_{t}^{Q}} \ln J_{P, i}^{Q}$.

\footnotetext{
${ }^{20}$ See Benth et al. (2008) for a discussion of the Girsanov transform for Poisson processes.
} 


\subsection{Option Price with Jumps}

Due to the inclusion of jumps, the logarithm of the underlying spot price is not normally distributed anymore and we cannot refer to the above derived option price formula. However, since the jump component is log-normally distributed, we can write the distribution of $\ln P_{t}$ conditional on the occurrence of $n$ jumps analogously to Merton (1976) as

$$
\ln P_{T} \mid n \text { Jumps } \sim \mathcal{N}\left(\frac{\ln P_{t}+\Delta f_{P}(\tau)+\left(\kappa_{P} \gamma^{Q}(\tau)-\frac{1}{2} \sigma_{P}^{2}\right) \tau+n \mu_{J}^{Q}}{\left(1+\tau \kappa_{P}\right)}, \frac{\sigma_{P}^{2} \tau+n \sigma_{P, J}^{2}}{\left(1+\tau \kappa_{P}\right)^{2}}\right),
$$

where again $\tau=T-t$ and $\Delta f_{P}(\tau)=f_{G}(T)-f_{G}(t)$. Therefore, the logarithm of the spot price conditional on the occurrence of a jump is again normally distributed. Thus, we can write the value of an option to exchange the electricity prices $G_{t}$ and $D_{t}$, allowing for jumps in $G_{t}$ and $D_{t}$, as the weighted sum of the option values without jumps. We only need to adjust the corresponding variances. ${ }^{21}$ The option price at time $t$ with maturity in $T$ can then be written as

$$
C_{t}\left(G_{t}, D_{t}\right)=\sum_{n=0}^{\infty} \frac{e^{-\lambda_{G}^{Q} t}\left(\lambda_{G}^{Q} t\right)^{n}}{n !} \sum_{m=0}^{\infty} \frac{e^{-\lambda_{D}^{Q} t}\left(\lambda_{D}^{Q} t\right)^{m}}{m !}\left(D_{t} \Phi\left(d_{1}^{J}\right)-G_{t} \Phi\left(d_{2}^{J}\right)\right),
$$

where $n(m)$ counts the number of jumps in $G_{t}\left(D_{t}\right)$ and the fractions correspond to the Poisson probabilities of a jump given the jump intensity $\lambda_{G}\left(\lambda_{D}\right)$. Finally, the values of $d_{1}^{J}$ and $d_{2}^{J}$ are given by

$$
\begin{aligned}
d_{1}^{J} & =\frac{\ln \left(\frac{D_{t}}{G_{t}}\right)+\frac{1}{2} \sigma^{2} \tau}{\sigma \sqrt{\tau}}, \\
d_{2}^{J} & =d_{1}^{J}-\sigma \sqrt{\tau}, \\
\sigma^{2} & =\frac{\sigma_{G}^{2}+n \sigma_{G, J}^{2}}{\left(1+\tau \kappa_{G}\right)^{2}}+\frac{\sigma_{D}^{2}+m \sigma_{D, J}^{2}}{\left(1+\tau \kappa_{D}\right)^{2}}-2 \rho \frac{\sqrt{\left(\sigma_{G}^{2}+n \sigma_{G, J}^{2}\right)\left(\sigma_{D}^{2}+m \sigma_{D, J}^{2}\right)}}{\left(1+\tau \kappa_{G}\right)\left(1+\tau \kappa_{D}\right)} .
\end{aligned}
$$

\section{Parameter Estimation}

\subsection{Deterministic Function}

In order to estimate the parameters of the stochastic component, we first need to filter out the deterministic function $f_{P}(t)$. We use a combination of a piecewise constant function and a sinusoidal function. Our deterministic function is based on those of

\footnotetext{
${ }^{21}$ Although the incorporation of jump also affects the mean value of $\ln P_{T}$, we neglect its adjustment since it has no impact on the value of a PTR option.
} 
Bierbrauer et al. (2007) and Seifert and Uhrig-Homburg (2007) and can be stated as

$$
f(t)=\alpha+\mu \cdot t+\mathbf{d} \cdot D_{d a y}+s_{1} \sin \left(\left(s_{2}+t\right) \frac{2 \pi}{365}\right) .
$$

Here, $t$ indicates the number of days (counting from 1 to 2,922). Further $\alpha, \mu, s_{1}, s_{2}$ are constant parameters, where $s_{1}$ and $s_{2}$ model the annual trend in electricity prices. $\mathbf{d}$ is a vector of seven parameters $\left(d_{\text {Mon }}-d_{\text {Sun }}\right)$ to model the weekly seasonality. $D_{\text {day }}$ is a vector of seven dummy variables $\left(D_{\text {Mon }}-D_{\text {Sun }}\right)$ where each entry takes the value of 1 if $t$ is the corresponding day of the week and 0 otherwise. We estimate all parameters using the least-squares method and perform the following calculation

$$
\min _{\alpha, \mu, s_{1}, s_{2}, \mathbf{d}} \sum_{t=1}^{T}\left(\ln P_{t}-f_{P}(t)\right)^{2} .
$$

The estimated values for the German and the Dutch electricity prices are shown in Table 3. All parameters refer to the logarithm of the spot price, where we only consider parameters which are significantly different from 0 at the $95 \%$ confidence level.

\section{[Insert Table 3 here]}

Table 3 shows the annual and intra-week seasonality of electricity prices which are comparable for both countries. While prices for weekdays are above average, with a peak around Tuesday through Thursday, weekend prices are below average, where prices on Sunday are the lowest. This pattern is intuitive and corresponds to intra-week demand patterns. The annual sinusoidal function is also comparable for both countries. Prices are generally higher during the fall and lower during the spring. This effect is more pronounced for the Netherlands but also apparent for Germany.

Having calculated the deterministic function, we receive the stochastic residual as the difference between the $\log$ spot price and the deterministic function. ${ }^{22}$ This stochastic component is used to estimate the parameters and latent state variables, i.e. jump times and jump sizes, of our model.

\subsection{Discrete Processes}

\subsubsection{Diffusion Model}

In order to estimate the parameters for our model, we first need to discretize the SDE of the stochastic component. Assuming $\Delta t=1$, we use the Euler discretization, i.e.

\footnotetext{
${ }^{22}$ We test the stationarity of the stochastic component using the Augmented Dickey-Fuller test. The existence of a unit root is rejected at the $1 \%$ level for German as well as for Dutch residuals. The corresponding t-value for the first is -8.8727 while for the latter the t-value is -9.2379 .
} 
$d S_{t}=S_{t}-S_{t-1} \cdot{ }^{23}$ Therefore, we can write the discrete processes of the stochastic component for our diffusion model as

$$
S_{t}=\left(1-\kappa_{P}\right) S_{t-1}+\sigma_{P} \varepsilon_{P}(t),
$$

where $\varepsilon_{P}(t) \stackrel{i i d}{\sim} \mathcal{N}(0,1)$, for $S_{t} \in\left\{X_{t}, Y_{t}\right\}$ and $P_{t} \in\left\{G_{t}, D_{t}\right\}$. Thus, $S_{t}$ has a conditional normal distribution, i.e.

$$
S_{t} \mid S_{t-1} \sim \mathcal{N}\left(\left(1-\kappa_{P}\right) S_{t-1}, \sigma_{P}^{2}\right)
$$

Based on the above stated conditional distribution, we can write the joint likelihood of $S=\left\{S_{t}\right\}_{t=1}^{T}$ as

$$
p(S \mid \Theta)=\prod_{t=1}^{T} p\left(S_{t} \mid S_{t-1}, \Theta\right),
$$

where $\Theta=\left(\kappa_{P}, \sigma_{P}^{2}, \rho\right)$ is the vector of parameters. Thus, the joint likelihood is just the product of the densities of $S_{t}$ for all $t=1, \ldots, T$.

\subsubsection{Jump-Diffusion Model}

When discretizing the SDE for the jump-diffusion model, we use the same Euler discretization as before. However, of special interest is the discretization of the jump component. In order to discretize the Poisson process, we use a series of Bernoulli distributed elements, i.e. $q_{t} \sim \mathcal{B}$ ernoulli $\left(\varphi_{t}\right)$, where $\varphi_{t}$ is the Bernoulli probability. Thus, the discretized process for $S_{t}$ is governed by

$$
S_{t}=\left(1-\kappa_{P}\right) S_{t-1}+\sigma_{P} \varepsilon_{P}(t)+\ln J_{P, t} q_{P, t},
$$

where $\varepsilon_{P}(t) \stackrel{\text { iid }}{\sim} \mathcal{N}(0,1)$. For the ease of notation we use $N_{t}=\ln J_{t}$ henceforth. The conditional distribution of $S_{t}$ is

$$
S_{t} \mid S_{t-1}, N_{t}, q_{t} \sim \mathcal{N}\left(\left(1-\kappa_{P}\right) S_{t-1}+N_{P, t} q_{P, t}, \sigma_{P}^{2}\right)
$$

which is still normal conditional on the occurrence of a jump. Since we condition on the appearance of a jump with known size, we do not need to adjust the variance but only the mean of the distribution of $S_{t}$. The joint likelihood of $S_{t}$ hence reads as

$$
p(S \mid \Theta, N, q)=\prod_{t=1}^{T} p\left(S_{t} \mid S_{t-1}, \Theta, N_{t}, q_{t}\right),
$$

\footnotetext{
${ }^{23}$ Eraker et al. (2003) show that the discretization bias is neglegible when using daily data.
} 
where $N=\left\{N_{t}\right\}_{t=1}^{T}$ and $q=\left\{q_{t}\right\}_{t=1}^{T}$. Here, the vector of parameters additionally includes the mean and variance of the jump size as well as the jump intensity, i.e. $\Theta=\left(\kappa_{P}, \sigma_{P}^{2}, \mu_{P, J}, \sigma_{P, J}^{2}, \lambda_{P, \rho}\right)$.

\subsection{Parameter Estimation}

\subsubsection{MCMC Method}

For the estimation of the parameters and latent state variables of our model, we use Markov Chain Monte Carlo (MCMC) estimation. Using MCMC we need to find the joint posterior distribution of our parameters and state variables, i.e. $p(\Theta, V \mid S)$, where $\Theta$ comprises all relevant parameters and $V$ represents the latent state variables, i.e. jump time and jump size in case of the jump-diffusion model. With this joint posterior distribution, we perform a Monte Carlo simulation in order to estimate the relevant parameters and state variables. As, however, the joint posterior distribution is in general not known, we make use of the Clifford-Hemmersley theorem. It states that the complete conditional distributions, i.e. $p(\Theta \mid S, V)$ and $p(V \mid S, \Theta)$, fully describe the joint posterior. Therefore, instead of drawing from the joint posterior, we can sample from the complete conditionals instead. If drawing from the complete conditionals is also not feasible, the Clifford-Hemmersley theorem can be reapplied until each parameter is sampled separately. The same holds for the complete conditional of the latent state variables. Drawing all relevant parameters and latent state variables iteratively we attain a Markov chain that eventually converges to the target posterior distribution. Using the chain of sample drawings, the point estimates of the parameters and latent state variables are obtained as the arithmetic mean of all Monte Carlo draws. We discard the first 10,000 iterations in order to allow the Markov Chain to reach a stationary distribution and use additional 10,000 iterations for estimating our parameters, thereby stabilizing our point estimates. ${ }^{24}$

MCMC is essentially based on the theory of Bayes. Applying Bayes rule, we can decompose the posterior distribution of parameters and state variables into the product of the joint likelihood, as stated above, the distribution of the state variables, $p(V \mid \Theta)$, and the prior distribution of the parameters, $p(\Theta)$. Since we only need a proportionality relation, the posterior distribution can be expressed as

$$
p(\Theta, V \mid P) \propto p(P \mid \Theta, V) p(V \mid \Theta) p(\Theta) .
$$

\footnotetext{
${ }^{24}$ For a textbook treatment of MCMC and Bayesian theory see Gamerman and Lopes (2006). Johannes and Polson (2003) provide a comprehensive discussion of the application of MCMC estimation for financial models.
} 
If we can directly sample from the above stated product, we use the so called Gibbs steps and randomly draw a new parameter sample for our Monte Carlo simulation. The ability to use a Gibbs step heavily depends on the chosen prior distribution of the parameters and state variables. Through adequate choices of the prior distributions, we mainly use Gibbs steps for both of our models. ${ }^{25}$ If the above stated product cannot be sampled directly, we need to refer to a Metropolis-Hastings step. Here, the product of the densities only needs to be evaluated numerically. In this case, a new sample candidate is drawn from a proposed density and then it is decided whether to accept this one as a draw from the target posterior according to a given acceptance criterion. We use the Metropolis-Hastings step when drawing samples for the correlation $\rho$ in both models.

\subsubsection{Diffusion Model}

When estimating the parameters of our models, we again refer to $S_{t} \in\left\{X_{t}, Y_{t}\right\}$ and $P_{t} \in\left\{G_{t}, D_{t}\right\}$ since for both countries the estimation procedure works identically. The only exception states the correlation $\rho$ where we need information from the German as well as the Dutch likelihood. ${ }^{26}$ Note that for the diffusion model we do not have latent state variables.

Starting with the mean-reversion speed of the electricity prices $\kappa_{P}$, the conditional posterior, assuming a normal prior, i.e. $p\left(\kappa_{P}\right) \sim \mathcal{N}\left(m_{P}, s_{P}^{2}\right)$, is

$$
p\left(\kappa_{P} \mid \Theta_{-\kappa_{P}}, S\right) \propto p(S \mid \Theta) p\left(\kappa_{P}\right) .
$$

$\Theta_{-\kappa_{P}}$ denotes the vector of parameters excluding $\kappa_{P}$. For the variance of the electricity prices we assume an inverse gamma prior, i.e. $p\left(\sigma_{P}^{2}\right) \sim \mathcal{I} \mathcal{G}\left(\alpha_{P}, \beta_{P}\right)$, in order to ensure non-negativity of the variance. The conditional posterior follows as

$$
p\left(\sigma_{P}^{2} \mid \Theta_{-\sigma_{P}^{2}}, S\right) \propto p(S \mid \Theta) p\left(\sigma_{P}^{2}\right) .
$$

Finally, we need to derive the conditional posterior of the correlation $\rho$. Since the correlation connects German and Dutch spot prices, the posterior of $\rho$ depends on the joint likelihood of both spot prices. Thus, the conditional posterior of $\rho$, assuming a uniform prior, i.e. $p(\rho) \sim \mathcal{U}\left(\rho_{\min }, \rho_{\max }\right)$ is

$$
p\left(\rho \mid \Theta_{-\rho}, X, Y\right) \propto p(X, Y \mid \Theta) p(\rho) .
$$

\footnotetext{
${ }^{25}$ In order to perform a Gibbs step, the prior distributions need to be conjugate to the likelihood. This means that the resulting posterior distribution is of the same family as the prior. For example, in case we assume an inverse-gamma prior and a normal likelihood, the resulting product is again inverse-gamma distributed from which we can directly smaple.

${ }^{26}$ We refer to the Appendix A.1 for details on the posterior distributions of the diffusion model.
} 
Since we cannot draw directly from this density, we use a random-walk MetropolisHastings step to draw samples for $\rho$. Here, we only need to evaluate $\pi\left(\rho_{g+1}\right) / \pi\left(\rho_{g}\right)$, where $\rho_{g}$ is the $g^{t h}$ sample drawing of $\rho$ and $\pi\left(\rho_{g}\right)$ is the posterior distribution of $\rho_{g}$. With random-walk Metropolis-Hastings, we draw a new candidate $\rho^{g+1}$, where $g+1$ is the next iteration drawing with $\rho^{g+1}=\rho^{g}+\varepsilon$ and $\varepsilon \sim \mathcal{N}\left(0, \sigma_{\varepsilon}^{2}\right)$. This draw is accepted with probability $\alpha$ given by ${ }^{27}$

$$
\alpha=\min \left(\frac{\pi\left(\rho^{g+1}\right)}{\pi\left(\rho^{g}\right)}, 1\right) .
$$

If $\rho^{g+1}$ is rejected as a draw from the target posterior distribution, we set $\rho^{g+1}=\rho^{g}$.

In Table 4 we present the parameters of the prior distributions (so called hyperparameters) alongside the starting values and estimation results for the diffusion model. Starting values are obtained as random draws from the prior distributions. We also provide information on the $95 \%$ confidence interval of estimated parameters given in brackets.

\section{[Insert Table 4 here]}

Results in Table 4 confirm the erratic behavior of electricity prices. The variance is relatively large compared to financial or other commodity markets and slightly higher in the Netherlands than in Germany. This result is in line with the differences in the power generation mix discussed above. The mean-reversion speed shows the short-term characteristics of jumps in electricity prices. Jumps generally fade within four days, where the mean-reversion speed is also higher for Dutch prices compared to German ones. Further, the correlation is also relatively high due to the ongoing convergence of electricity markets in Europe. The standard errors are rather small in relation to the parameter values indicating a fast convergence of our MCMC algorithm. Overall, the parameter values in Table 4 are in line with our expectations and the distributional characteristics shown in Table 1 and Figure 1.

\subsubsection{Jump-Diffusion Model}

In order to estimate the parameters and latent state variables for the jump-diffusion model we start with constructing a vector of jump times, i.e. $q_{t} \in\{0,1\}$ for $t=1, \ldots, T$.

\footnotetext{
${ }^{27}$ Generally, the acceptence criterion also contains the proposal densities of the correlation. However, since we use the random-walk Matropolis-Hastings step, the proposal distributions cancel out and we refrain from including them in the acceptance criterion.
} 
Each element of this vector be Bernoulli distributed, i.e. ${ }^{28}$

$$
q_{t} \mid S_{t-1}, \Theta, N_{t}, q_{t} \sim \mathcal{B} \text { ernoulli }\left(\varphi_{t}\right)
$$

$\varphi_{t}$ is the Bernoulli probability, i.e. the probability of a jump at time $t$. Using this vector of jump times, we draw the new jump intensity $\lambda_{P}$. We assume a beta prior for the jump intensity, i.e. $\lambda_{P} \sim \mathcal{B}$ eta $\left(\alpha_{\lambda}, \beta_{\lambda}\right)$, to ensure that the intensity is bounded by 0 and 1. Moreover, the beta prior allows us to apply a Gibbs step for $\lambda_{P}$. The posterior distribution follows as

$$
p\left(\lambda_{P} \mid q\right) \propto p\left(q \mid \lambda_{P}\right) p\left(\lambda_{P}\right) .
$$

We omit indices for jump times and jump sizes in case we refer to the entire vector. This is applicable to the posterior distributions of the parameters. When drawing the state variables, however, we apply indices as we sample each element of the vector separately.

Following the jump intensity, we need to generate a vector of jump sizes $N$. Since every element of this vector is normally distributed, i.e. $N_{t} \sim \mathcal{N}\left(\mu_{J}, \sigma_{J}^{2}\right)$, we can write the conditional posterior as

$$
p\left(N_{t} \mid \Theta, S_{t}, S_{t-1}, q_{t}\right) \propto p\left(S_{t} \mid S_{t-1}, \Theta, N_{t}, q_{t}\right) p\left(N_{t} \mid \Theta\right) .
$$

Since the product is also normally distributed, we again use a Gibbs step when drawing the vector of jump sizes. Having completed the draws for the jump times and jump sizes as well as the jump intensity, we continue by drawing all remaining parameters. First, we sample the mean jump size $\mu_{J}$ assuming a normal prior, i.e. $\mu_{J} \sim \mathcal{N}\left(m_{\mu}, s_{\mu}^{2}\right)$. Therefore, the posterior distribution is

$$
p\left(\mu_{J} \mid \Theta, N, q\right) \propto p(N \mid \Theta) p\left(\mu_{J}\right)
$$

which again is normal and thus, we also apply a Gibbs step. For the variance of the jump size we assume, in line with the variance of the diffusion process above, an inversegamma prior, i.e. $\sigma_{J}^{2} \sim \mathcal{I} \mathcal{G}\left(\alpha_{J}, \beta_{J}\right)$. The posterior distribution is also inverse-gamma and follows as

$$
p\left(\sigma_{J}^{2} \mid \Theta, N, q\right) \propto p(N \mid \Theta) p\left(\sigma_{J}^{2}\right) .
$$

The posterior distributions for the other parameters, i.e. mean-reversion speed, diffusion variance and correlation are obtained analogously to the diffusion model. We only condition each parameter on the vector of jump times and jump sizes. We refrain from stating the posterior distributions again and refer to the appendix.

\footnotetext{
${ }^{28}$ We refer to Appendix A.2 for details on the posterior distributions of the jump-diffusion model.
} 
All prior distributions and hyperparameters for the jump-diffusion model are presented in Table 5. We also state the starting values, which are again drawn as random samples from the prior distributions. The starting vector for the jump sizes is constructed based on observed market data. Further, the estimated values for the parameters as well as their $95 \%$ confidence intervals (given in brackets) are shown.

\section{[Insert Table 5 here]}

Table 5 displays quite similar results for Germany and the Netherlands. The meanreversion speed as well as the diffusion variance significantly decrease compared to Table 4 due to the inclusion of jumps. The mean jump size is slightly negative for both countries, however negligible in relation to the large variance of the jump size. The jump intensity indicates around 35 jumps in Germany and 45 in the Netherlands on average per year. The higher jump intensity as well as the higher jump size variance observed for Dutch electricity prices are in line with the power generation mix discussed above. Further, the correlation declines compared to the diffusion model since we now include uncorrelated jumps in both prices. Overall, the results of our parameter estimation also confirm the time-series results from Table 1 and Figure 1.

\subsubsection{Risk-Neutral Parameters}

When deriving the risk-neutral parameters, we first of all only consider those parameters altered when changing to the risk-neutral measure $\mathcal{Q}$. Further, we only need to change those ones that influence the option price. Since both option pricing formulas, i.e. diffusion and jump-diffusion, are independent of the drift component, the market price of diffusion risk as well as the mean jump size do not affect PTR option prices. Therefore, only the risk-neutral jump intensity $\lambda_{P}^{Q}$ is to be estimated. In order to do so for the risk-neutral jump intensities for Germany and the Netherlands, we minimize, according to Broadi et al. (2007), the squared error between model and market prices. ${ }^{29}$ Thus, we carry out the following calculation

$$
\min _{\lambda_{P}^{Q}} \sum_{i=1}^{N}\left(C_{i}\left(G_{i}, D_{i}\right)-P T R_{i}\right)^{2} .
$$

Here, $C_{i}\left(G_{i}, D_{i}\right)$ is the model price of the monthly PTR option, $P T R_{i}$ is the observed market price and $N$ is the number of total PTR prices available. The results of the above indicated calculation show, that in order to minimize the squared error between

${ }^{29}$ In contrast to our approach, Broadi et al. (2007) minimize the difference between model and market Black/Scholes implied volotilities in order to prevent placing too much weight on expensive options. In our case, however, this approach is not feasible since implied volatilites of the Margrabe model are not available for all monthly PTR options. 
model and market PTR prices, both jump intensities tend towards zero, i.e. $\lambda_{P}^{Q} \rightarrow 0$, for all $P \in\{G, D\} .^{30}$

The effect of decreasing jump intensities on PTR option prices is shown in Figure 3. It plots the arithmetic mean of the model prices against the jump intensities of German and Dutch electricity prices.

\section{[Insert Figure 2 here]}

Figure 2 shows that PTR prices are strictly increasing in both parameters despite the negative mean jump size. As, however, the mean jump size does not enter into the PTR option price but only the jump size variance, this result is intuitive. The average model price approaches 8.80 EUR in case both jump intensities tend towards zero. Considering the average market price of about 7.90 EUR from Table 2, Figure 2 indicates that even without jumps in the underlying asset prices, the model overestimates the resulting option values.

\subsection{De-Spiked Process}

Besides analyzing the impact of including jumps in a valuation model for monthly PTR options, we are also interested in the extent to which underlying electricity prices are an adequate input factor for those models. Since there is only a loose connection between intertemporal electricity prices, using observed prices for the valuation of electricity options could lead to sizeable distortions. This risk is especially apparent in the presence of extreme jumps in the underlying electricity prices. As electricity prices are mean-reverting, jumps fade usually within a few days. ${ }^{31}$ For the valuation of an option on electricity prices, or on the spread as in our case, with several days to maturity, only the overall price level should be of interest. Thus, we analyze whether the pricing performance of our models is improved when considering only de-spiked prices. These are simply the observed electricity prices adjusted by extreme jumps, i.e. the diffusion component of the underlying electricity price.

We de-spike our process again using the method of MCMC, or rather exploiting the already performed estimation outcome. Via MCMC we receive a vector of jump times and jump sizes during each iteration. The corresponding vector of de-spiked prices then simply results as the difference between observed market prices and the product of

\footnotetext{
${ }^{30} \mathrm{~A}$ jump intensity of exactly zero is not feasible since the Poisson probability for a jump intensity of zero cannot be calculated.

${ }^{31}$ Seifert and Uhrig-Homburg (2007) for example show that jumps in German electricity prices only last about two to three days.
} 
jump times and jump sizes. The final vector of de-spiked prices is then calculated analogously to the parameter estimates as the arithmetic mean of all non-discarded iterations. Figure 3 shows the de-spiked logarithm of daily baseload prices for Germany and the Netherlands between 2001 and 2008.

\section{[Insert Figure 3 here]}

\section{Empirical Results}

In this section we compare the pricing performance of the four models discussed above regarding their ability to match observed monthly PTR option prices. Besides implementing the diffusion and jump-diffusion model, we distinguish for each of them between using observed or de-spiked prices. Our analysis is based on 95 monthly PTR options between February 2001 and December $2008 .^{32}$ The time to maturity of each PTR, given in years, is calculated as the difference between the day of the auction and the first day of the delivery period. ${ }^{33}$ Table 6 shows the descriptive statistics and the Root Mean Squared Error (RMSE) of the spread between model and market PTR prices. A negative difference indicates a model price exceeding the respective market price and vice versa.

\section{[Insert Table 6 here]}

The results in Table 6 show two effects. First of all, including jumps in the underlying process significantly enhances the pricing performance of our model. This effect is evident for the regular as well as the de-spiked price processes. Second, using de-spiked prices also improves the pricing performance of both the diffusion and the jump-diffusion model. Although the former effect reduces the RMSE between model and market PTR prices more strongly, the latter one is still significant. In order to test the significance of the inclusion of jumps as well as the usage of de-spiked prices, we refer to the LikelihoodRatio test applied, among others, by Longstaff et al. (2001). We find that effects emphasized in Table 6 are significant at the $99 \%$ confidence level. While the test statistics for the comparison of the diffusion and the jump-diffusion model is 109.85 , the corresponding values for the diffusion and jump-diffusion model based on de-spiked prices in contrast to observed prices are 14.44 and 47.45 respectively. ${ }^{34}$

\footnotetext{
${ }^{32}$ The January 2001 PTR option is not included in our analysis. Since there are not electricity spot prices available for December 2000, we are not able to calculate the corresponding model price.

${ }^{33}$ Our calculations are based on a constant interest rate of $2 \%$ p.a. Due to the relatively short time to maturity, however, the resulting option prices are highly insensitive towards changes in the interest rate.

${ }^{34}$ As we only observe one market price per PTR option, our Likelihood-Ratio test is based on the $\chi^{2}$ distribution with $1 \mathrm{df}$.
} 
Using de-spiked prices leads to a decrease in overall PTR model prices as indicated by the higher mean values of the spread. The largest effect, however, is evident for the minimum values of the spread between model and market PTR prices. A large negative value (in absolute terms) indicates PTR model prices significantly above observed market prices. These extreme model prices are based on jumps in the underlying spot price. Since despiking eliminates those outliers, exaggerated model prices are also reduced. As jumps are usually positive, using de-spiked prices results in generally lower values for model PTRs and thus, in a better fit of PTR market prices.

The effect of the inclusion of jumps is similar. Table 5 shows that the diffusion variance markedly declines for the jump-diffusion model compared to the diffusion model. Considering the risk-neutral jump intensities of nearly zero, only the diffusion variance enters into the valuation of PTR options. Hence, the overall PTR model price decreases as it is strictly increasing in the variance, leading to a better fit of market prices. The effect of reduced spread values is stressed by the mean and median as well as the minimum and maximum values in Table 6 .

In addition to an improved model performance, results in Table 6 point out that PTR model prices are on average above their market counterparts as indicated by the negative mean spread values, i.e. monthly PTR options are generally underpriced. Even for the jump-diffusion model with jump intensities of virtually zero, model prices are above market prices. ${ }^{35}$ In order to confirm the undervaluation of monthly PTRs, we compare their prices to those of the corresponding futures spread. Since a PTR is an option on the spread between the German and Dutch electricity spot price, the corresponding futures contract is the spread between the German and Dutch national electricity futures with identical delivery period. In our case, we use a long position in the Dutch and a short position in the German futures contract. Further, we use physically settled futures contracts from the EEX, i.e. German Power Futures, and the ENDEX, i.e. Dutch Power Futures, in order to match the physical settlement of PTRs. The calculation of the futures spread is based on the daily closing price at the day of the PTR auction. Figure 4 shows the price of the monthly PTR option (solid line) and the corresponding spread between the national electricity futures contracts (dashed line) for all contracts between February 2006 and December 2008.

\section{[Insert Figure 4 here]}

\footnotetext{
${ }^{35}$ Even though monthly PTR options are rather portfolios of hourly PTR options, we value these contracts as plain-vanilla options with expiry on the first day of the delivery period. The valuation of monthly PTR options as a portfolio of hourly options would, however, entail an increase in model prices as the time to maturity generally increases. Therefore, overestimated PTR model prices and thus the underpricing of market PTRs would be even more pronounced.
} 
Figure 4 shows that PTR prices and the corresponding futures spreads are highly correlated. However, almost all of the monthly PTR option prices are below the corresponding futures spread. ${ }^{36}$ While PTR contracts offer the flexibility of exercising every hourly PTR contract separately, futures contracts in contrast pose a binding commitment over the entire delivery period. Thus, the flexibility inherent in PTRs is not priced supporting our results from Table 6 that monthly PTR options are generally undervalued. This is in line with the prior findings of Dieckmann et al. (2008).

\section{Conclusion}

Cross-border electricity flows become increasingly relevant due to connecting pan European electricity markets. Further, shifts in the power generation mix, i.e. a growing share of renewables along with a decrease of fossil fuels and nuclear power, result in additional cross-border electricity flows. These trends increase the physical and financial risk inherent in cross-border electricity markets. Although TSOs continue to invest in the expansion of their intra- and international power grids, congestion will continue to prevail in the European electricity markets.

Physical Transmission Rights (PTRs) are physically settled option contracts in order to manage the capacity of cross-border interconnections in Europe. These products are currently the only used instruments for managing electricity flows at German crossborder interconnections. From a financial perspective, PTRs are options to exchange one electricity price for another. We therefore apply the exchange option model of Margrabe (1978) for the valuation of monthly PTR contracts on the German and Dutch interconnector between 2001 and 2008. We extend the Margrabe model by including jumps in the underlying pice process in order to model the erratic behavior of electricity prices. Our analysis shows that the adoption of jumps significantly improves the pricing performance of the exchange option model. In addition, we also show that using de-spiked prices further improves the performance for both, the diffusion as well as the jump-diffusion model. These two effects are in line with our expectations. As electricity prices are extremely erratic compared to other commodities, their distribution is far from normal even for monthly average values. Thus, including jumps in the model accommodates the non-normal distribution and clearly improves the ability of the model to match PTR market prices. Further, as intertemporal electricity prices are at best loosely connected, de-spiked prices are a better indicator of the overall price level and a possible bias induced by jumps in the underlying is significantly reduced.

\footnotetext{
${ }^{36}$ Results from Figure 4 are also apparent when using settlement prices from the day prior to the auction of monthly PTR options.
} 
In addition to the pricing performance of the discussed models, numerical results show that monthly PTR options are generally underpriced. This underpricing is not only evident in relation to calculated model prices, but also compared to the corresponding futures spread. For contracts between February 2006 and December 2008, the PTR option price is strictly below the respective futures spread indicating that market participants do not value the flexibility inherent in PTR options. This result confirms the prior findings of Dieckmann et al. (2008).

Future research could extend our analysis and apply our model to additional spread options. Here, spread products in other commodity markets are of special interest. These include locational spreads as well as cross commodity contracts, such as the widely used spark-spread options. In addition, a thorough analysis of the difference between exchange options in contrast to options directly written on the underlying spread are of great interest. 


\section{Appendix}

\section{A.1 Posterior Densities for the Diffusion Model}

The conditional posterior for the mean reversion speed is

$$
\begin{aligned}
p\left(\kappa_{P} \mid \Theta_{-\kappa_{P}}, S\right) & \propto p(S \mid \Theta) p\left(\kappa_{P}\right) \\
& \propto \exp \left(-\frac{\left(\kappa_{P}-\frac{s_{P}^{2} \sum_{t=1}^{T} S_{t-1}^{2}-s_{P}^{2} \sum_{t=1}^{T} S_{t} S_{t-1}+\sigma_{P}^{2} m_{P}}{s_{P}^{2} \sum_{t=1}^{T} S_{t-1}^{2}+\sigma_{P}^{2}}\right)^{2}}{2 \frac{\sigma_{P}^{2} s_{P}^{2}}{s_{P}^{2} \sum_{t=1}^{T} S_{t-1}^{2}+\sigma_{P}^{2}}}\right) .
\end{aligned}
$$

The conditional posterior for the variance of the diffusion process reads as

$$
\begin{aligned}
p\left(\sigma_{P}^{2} \mid \Theta_{-\sigma_{P}^{2}}, S\right) & \propto p(S \mid \Theta) p\left(\sigma_{P}^{2}\right) \\
& \propto \frac{\widetilde{\beta}_{P}}{\Gamma\left(\widetilde{\alpha}_{P}\right)}\left(\frac{1}{\sigma_{P}^{2}}\right)^{\widetilde{\alpha}_{P}+1} \exp \left(-\frac{\widetilde{\beta}_{P}}{\sigma_{P}^{2}}\right) .
\end{aligned}
$$

Therefore the posterior of $\sigma_{P}^{2}$ is also inverse-gamma distributed where the parameters follow from the prior parameters as

$$
\begin{aligned}
& \alpha_{P}=\frac{1}{2} T+\alpha_{P}, \\
& \beta_{P}=\frac{1}{2} \sum_{t=1}^{T}\left(S_{t}-\left(1-\kappa_{P}\right) S_{t-1}\right)^{2}+\beta_{P} .
\end{aligned}
$$

The conditional posterior for the correlation between German and Dutch electricity prices is

$$
\begin{aligned}
p\left(\rho \mid \Theta_{-\rho}, X, Y\right) \propto & p(X, Y \mid \Theta) p(\rho) \\
\propto & \frac{1}{|\Sigma|^{\frac{T}{2}}} \exp \left(-\frac{1}{2} \sum_{t=1}^{T}\left(\mathbf{S}_{t}-\boldsymbol{\mu}_{t-1}\right)^{\prime} \Sigma^{-1}\left(\mathbf{S}_{t}-\boldsymbol{\mu}_{t-1}\right)\right) \\
& \cdot \frac{1}{\rho_{\max }-\rho_{\min }},
\end{aligned}
$$

where $\mathbf{S}_{t}=\left(X_{t}, Y_{t}\right)^{\prime}$ is the vector of de-trended spot prices. The vector of the mean of de-trended spot prices, i.e. $\boldsymbol{\mu}_{t-1}$, is $\boldsymbol{\mu}_{t-1}=\left(\left(1-\kappa_{G}\right) X_{t-1},\left(1-\kappa_{D}\right) Y_{t-1}\right)^{\prime} . \Sigma$ is the covariance matrix. 


\section{A.2 Posterior Densities for the Jump-Diffusion Model}

The Bernoulli probability $\varphi_{t}$ satisfies

$$
\begin{aligned}
\varphi_{t} & =\operatorname{Pr}\left(\varphi_{t}=1 \mid S_{t-1}, \Theta, N_{t}, q_{t}\right) \\
& =\left(1+\frac{1-\lambda_{P}}{\lambda_{P}} \exp \left(-\frac{2 N_{t}\left(S_{t}-\left(1-\kappa_{P}\right) S_{t-1}\right)-N_{t}^{2}}{\sigma_{P}^{2}}\right)\right)^{-1} .
\end{aligned}
$$

The posterior of the jump intensity follows as

$$
\begin{aligned}
p\left(\lambda_{P} \mid q\right) & \propto p\left(q \mid \lambda_{P}\right) p\left(\lambda_{P}\right) \\
& \propto \lambda_{P}^{\sum_{t=0}^{T} q_{t}}\left(1-\lambda_{P}\right)^{T-\sum_{t=0}^{T} q_{t}} \lambda_{P}^{\alpha_{\lambda}-1}\left(1-\lambda_{P}\right)^{\beta_{\lambda}-1} \\
& \propto \lambda_{P}^{\alpha_{\lambda}+\sum_{t=0}^{T} q_{t}-1}\left(1-\lambda_{P}\right)^{\beta_{\lambda}+T-\sum_{t=0}^{T} q_{t}-1} .
\end{aligned}
$$

We can write the conditional posterior of the mean jump size as

$$
\begin{aligned}
p\left(N_{t} \mid \Theta, S_{t}, S_{t-1}, q_{t}\right) & \propto p\left(S_{t} \mid S_{t-1}, \Theta, N_{t}, q_{t}\right) p\left(N_{t} \mid \Theta\right) \\
& \propto \exp \left(-\frac{1}{2} \frac{\left(N_{t}-\frac{\sigma_{J}^{2} q_{t}\left(S_{t}-\left(1-\kappa_{P}\right) S_{t-1}\right)+\sigma_{P}^{2} \mu_{J}}{\sigma_{J}^{2} q_{t}^{2}+\sigma_{P}^{2}}\right)^{2}}{\frac{\sigma_{J}^{2} \sigma_{P}^{2}}{\sigma_{J}^{2} q_{t+1}^{2}+\sigma_{P}^{2}}}\right) .
\end{aligned}
$$

The posterior distribution of the mean jump size is

$$
\begin{aligned}
p\left(\mu_{J} \mid \Theta, N, q\right) & \propto p(N \mid \Theta) p\left(\mu_{J}\right) \\
& \propto \exp \left(-\frac{1}{2} \frac{\left(\mu_{J}-\frac{s_{\mu}^{2} \sum_{t=1}^{T} N_{t}+\sigma_{J}^{2} m_{\mu}}{s_{\mu}^{2} T+\sigma_{J}^{2}}\right)^{2}}{\frac{\sigma_{J}^{2} s_{\mu}^{2}}{s_{\mu}^{2} T+\sigma_{J}^{2}}}\right) .
\end{aligned}
$$

The posterior distribution of the variance of the jump size follows as

$$
\begin{aligned}
p\left(\sigma_{J}^{2} \mid \Theta, N, q\right) & \propto p(N \mid \Theta) p\left(\sigma_{J}^{2}\right) \\
& \propto\left(\frac{1}{\sigma_{J}^{2}}\right)^{\frac{1}{2} T+\alpha_{J}+1} \exp \left(-\frac{\frac{1}{2} \sum_{t=0}^{T-1}\left(N_{t+1}-\mu_{J}\right)^{2}+\beta_{J}}{\sigma_{J}^{2}}\right) .
\end{aligned}
$$

The posterior distribution of the mean reversion speed is

$$
\begin{aligned}
p\left(\kappa_{P} \mid \Theta, S, N, q\right) & \propto p(S \mid \Theta, N, q) p\left(\kappa_{P}\right) \\
& \propto \exp \left(-\frac{\left(\kappa_{G}-\frac{\sigma_{P}^{2} m_{P}-s_{P}^{2} \sum_{t=1}^{T} S_{t-1}\left(S_{t}-S_{t-1}-N_{t} q_{t}\right)}{s_{P}^{2} \sum_{t=1}^{T} S_{t-1}^{2}+\sigma_{P}^{2}}\right)^{2}}{2 \frac{\sigma_{P}^{2} s_{P}^{2}}{s_{P}^{2} \sum_{t=1}^{T} S_{t-1}^{2}+\sigma_{P}^{2}}}\right) .
\end{aligned}
$$


The posterior distribution of the variance of the diffusion process follows as

$$
\begin{aligned}
p\left(\sigma_{P}^{2} \mid \Theta, N, q\right) & \propto p(S \mid \Theta, N, q) p\left(\sigma_{P}^{2}\right) \\
& \propto\left(\frac{1}{\sigma_{P}^{2}}\right)^{\frac{T}{2}+\alpha_{P}+1} \exp \left(-\frac{\frac{1}{2} \sum_{t=1}^{T}\left(S_{t}-\left(1-\kappa_{P}\right) S_{t-1}-N_{t} q_{t}\right)^{2}+\beta_{P}}{\sigma_{P}^{2}}\right) .
\end{aligned}
$$

The conditional posterior of the correlation is

$$
\begin{aligned}
p\left(\rho \mid \Theta_{-\rho}, X, Y, N^{G}, q^{G}, N^{D}, q^{D}\right) \propto & p\left(X, Y \mid \Theta, N^{G}, q^{G}, N^{D}, q^{D}\right) p(\rho) \\
\propto & \frac{1}{|\Sigma|^{\frac{T}{2}}} \exp \left(-\frac{1}{2} \sum_{t=1}^{T}\left(\mathbf{S}_{t}-\boldsymbol{\mu}_{t-1}\right)^{\prime} \Sigma^{-1}\left(\mathbf{S}_{t}-\boldsymbol{\mu}_{t-1}\right)\right) \\
& \cdot \frac{1}{\rho_{\max }-\rho_{\min }},
\end{aligned}
$$

where $\mathbf{S}_{t}=\left(X_{t}, Y_{t}\right)^{\prime}$ is the vector of spot prices. The vector of mean spot prices, i.e. $\boldsymbol{\mu}_{t-1}$, is defined as $\boldsymbol{\mu}_{t-1}=\left(\left(1-\kappa_{G}\right) X_{t-1}-N_{t}^{G} q_{t}^{G},\left(1-\kappa_{D}\right) Y_{t-1}-N_{t}^{D} q_{t}^{D}\right)^{\prime} . \Sigma$ is the covariance matrix. 


\section{References}

Albizzati, M.O., and Geman, H. (1994). Interest Rate Risk Management and Valuation of the Surrender Option in Life Insurance Policies. The Journal of Risk and Insurance, 61, 616-637.

Armstrong, M., and Galli, A. (2005). Are Day-Ahead Prices for Electricity Converging in Continental Europe? Working Paper, Cerna.

Benth, F.E., Benth, J.Š, and Koekebakker, S. (2008). Stochastic Modelling of Electricity and Related Markets. World Scientific.

Bierbrauer, M., Menn, C., Rachev, S.T., and Trück, S. (2007). Spot and Derivative Pricing in the EEX Power Market. Journal of Banking \& Finance, 31, 3462-3485.

Broadi, M., Chernov, M., and Johannes, M. (2007). Model Specification and Risk Premia: Evidence from Futures Options. Journal of Finance, 62, 1453-1490.

Carr, P. (1988). The Valuation of Sequential Exchange Options. Journal of Finance, $43,1235-1256$.

Cartea, A., and Figueroa, M.G. (2005). Pricing in Electricity Markets: A Mean Reverting Jump Diffusion Model with Seasonality. Applied Mathematical Finance, 12, 313-335.

Cherubini, U., and Luciano, E. (2002). Bivariate Option Pricing with Copulas. Applied Mathematical Finance, 9, 69-85.

Cont, R., and Tankov, P. (2003). Financial Modelling with Jump Processes. Chapman $\& \mathrm{Hall} / \mathrm{CRC}$.

Dempster, M.A.H., Medova, E., and Tang, K. (2008). Long Term Spread Option Valuation and Hedging. Journal of Banking \& Finance, 32, 2530-2540.

Dieckmann, B., Reichmann, O., and Wobben, M. (2008). Bewertung von Physischen Übertragungsrechten - Eine Analyse der Grenzkuppelstellen zwischen Deutschland und den Niederlanden. Zeitschrift für Energiewirtschaft, 4, 254-261.

Eraker, B. (2004). Do Stock Prices and Volatility Jump? Reconciling Evidence from Spot and Option Prices. Journal of Finance, 59, 1367-1403.

Eraker, B., Johannes, M., and Polson, N. (2003). The Impact of Jumps in Volatility and Returns. Journal of Finance, 63, 1269-1300.

Fischer, S. (1978). Call Option Pricing When the Exercise Price is Uncertain, and the Valuation of Index Bonds. Journal of Finance, 33, 169-176. 
$\mathrm{Fu}$, Q. (1996). On the Valuation of an Option to Exchange One Interest Rate for Another. Journal of Banking \& Finance, 20, 645-653.

Gamerman, D., and Lopes, H.F. (2006). Markov Chain Monte Carlo - Stochastic Simulation for Bayesian Inference. Chapman \& Hall/CRC.

Gastineau, G. (1993). An Introduction to Special-Purpose Derivatives: Options with a Payout Depending on More than One Variable. Journal of Derivatives, 1, 98-104.

Gay, G.D., and Manaster, S. (1984). The Quality Option Implicit in Futures Contracts. Journal of Financial Economics, 13, 353-370.

Gerber, H.U., and Shiu, E.S.W. (1996). Martingale Approach to Pricing Perpetual American Options on Two Stocks. Mathematical Finance, 6, 303-322.

Girsanov, I.V. (1960). On Transforming a Certain Class of Stochastic Processes by Absolutely Continuous Substitution of Measures. Theory of Probability and its Applications, 5, 285-301.

Grinblatt, M., and Titman, S. (1989). Adverse Risk Incentives and the Design of Performance-Based Contracts. Management Science, 35, 807-822.

Hemler, M.L. (1990). The Quality Option in Treasury Bond Futures Contracts. Journal of Finance, 45, 1565-1586.

Johannes, M., and Polson, N. (2003). MCMC Methods for Continuous-Time Financial Econmetrics. Working Paper, University of Chicago.

Johnson, S.A., and Tian, Y.S. (2000). Indexed Executive Stock Options. Journal of Financial Economics, 57, 35-64.

Kristiansen, T. (2004). Congestion Management, Transmission Pricing and Area Price Hedging in the Nordic Region. Electrical Power and Energy Systems, 26, 685-695.

Li, M. (2008). The Impact of Return Nonnormality on Exchange Options. Journal of Futures Markets, 9, 845-870.

Longstaff, F.A., Santa-Clara, P., and Schwartz, E.S. (2001). The Relative Valuation of Caps and Swaptions: Theory and Empirical Evidence. Journal of Finance, 56, 2067-2109.

Lucia, J.J., and Schwartz, E.S. (2002). Electricity Prices and Power Derivatives: Evidence from the Nordic Power Exchange. Review of Derivatives Research, 5, 5-20. 
Marckhoff, J., and Muck, M. (2009). Jump Risk Premia in Short-Term Spread Options: Evidence from the German Electricity Market. Working Paper, University of Bamberg.

Marckhoff, J., and Wimschulte, J. (2009). Locational Price Spreads and the Pricing of Contracts for Difference: Evidence from the Nordic Market. Energy Economics, 31, $257-268$.

Margrabe, W. (1978). The Value of an Option to Exchange One Asset for Another. Journal of Finance, 23, 177-186.

Margrabe, W. (1993). Triangular Equilibrium and Arbitrage in the Market for Options to Exchange Two Assets. Journal of Derivatives, 1, 60-69.

Merton, R.C. (1976). Option Pricing when Underlying Stock Returns are Discontinuous. Journal of Financial Economics, 3, 125-144.

Mikosch, T. (1999). Elementary Stochastic Calculus. World Scientific.

Rodrigues, P.J.M., and Schlag, C. (2009). A Jumping Index of Jumping Stocks? An MCMC Analysis of Continuous-Time Models for Individual Stocks. Working Paper, House of Finance, Goehte University.

Seifert, J., and Uhrig-Homburg, M. (2007). Modelling Jumps in Electricity Prices: Theory and Empirical Evidence. Journal of Derivatives Research, 10, 59-85.

Von der Fehr, N.H.M., Amundsen, E.S., and Bergman, L. (2005). The Nordic Market: Signs of Stress? The Energy Journal, 26 (Special Issue), 71-98.

Zachmann, G. (2008). Electricity Wholesale Market Prices in Europe: Convergence? Energy Economics, 30, 1659-1671. 
Table 1

Descriptive Statistics of Daily and Monthly Spot Prices

This table shows the descriptive statistics of the logarithm of daily (Section A) and monthly (Section B) day-ahead electricity prices from Germany and the Netherlands. Calculated values in Section A are based on 2,922 daily prices between January 1, 2001 and December 31, 2008. Results in Section B are based on the 96 monthly average electricity prices between 2001 and 2008. Monthly averages are calculated as the arithmetic mean of the logarithm of all daily prices per calendar month.

$\begin{aligned} & \text { A. Daily Baseload } \\
& \text { Country }\end{aligned}$
\begin{tabular}{lrrrrrrr} 
Mean & Median & Minimum & Maximum & Std.dev. & Skewness & Kurtosis \\
\hline Germany & 3.5086 & 3.4667 & 1.1378 & 5.7089 & 0.5110 & 0.0623 & 0.4531 \\
Netherlands & 3.6690 & 3.6112 & 0.7178 & 6.4928 & 0.5273 & 0.2431 & 1.0480
\end{tabular}

B. Monthly Average

\begin{tabular}{lrrrrrrr} 
Country & Mean & Median & Minimum & Maximum & Std.dev. & Skewness & Kurtosis \\
\hline Germany & 3.5090 & 3.4028 & 2.8299 & 4.4675 & 0.4052 & 0.4328 & -0.7689 \\
Netherlands & 3.6689 & 3.5694 & 2.9725 & 4.4766 & 0.3784 & 0.3439 & -0.9334 \\
\hline
\end{tabular}


Table 2

Descriptive Statistics of Monthly PTR Options

This table shows the descriptive statistics for the 96 monthly PTR options on the German-Dutch interconnector between January 2001 and December 2008.

\begin{tabular}{lrrrrrrrr}
\hline \hline PTR & N & Mean & Median & Minimum & Maximum & Std.dev. & Skewness & Kurtosis \\
\hline Month & 96 & 7.8988 & 6.8850 & 1.0620 & 27.8886 & 5.3103 & 1.5583 & 3.3452 \\
\hline \hline
\end{tabular}


Table 3

Parameters of Deterministic Function

This table shows the estimated values of the deterministic functions for Germany and the Netherlands between 2001 and 2008. Calculations are based on the logarithm of daily prices. All parameters are significantly different from 0 at the $95 \%$ level.

\begin{tabular}{lcccccccccc}
\hline \hline Country & $\alpha$ & $\mu$ & $d_{\text {Mon }}$ & $d_{\text {Tue }}$ & $d_{\text {Wed }}$ & $d_{\text {Thu }}$ & $d_{\text {Fri }}$ & $d_{\text {Sat }}$ & $d_{\text {Sun }}$ & $s_{1}$ \\
\hline Germany & 2.9415 & 0.0004 & 0.0896 & 0.1598 & 0.1513 & 0.1293 & 0.0667 & -0.1645 & -0.4332 & -0.0494 \\
Netherlands & 3.1985 & 0.0003 & 0.1044 & 0.1653 & 0.1429 & 0.1320 & 0.0629 & -0.1678 & -0.4407 & -0.1010 \\
\hline \hline
\end{tabular}


Table 4

Estimated Parameters and Prior Distributions for the Diffusion Model

This table provides information on the parameters of the diffusion model. It shows the prior distributions and their respective hyperparameters. Further, the starting value for each parameter is given which is obtained as a random draw from its prior distribution. Finally, the point estimates and standard errors (given in brackets) for the parameters are shown calculated as the arithmetic mean of all non-discarded iterations.

\begin{tabular}{lrrr}
\hline \hline Parameter & Prior Distribution & Starting Values & Estimated Values \\
\hline$\kappa_{G}$ & $\mathcal{N}(0.5,0.25)$ & $\kappa_{G,(0)}=0.8639$ & $0.2291(0.00012)$ \\
$\sigma_{G}^{2}$ & $\mathcal{I} \mathcal{G}(3.0,1.0)$ & $\sigma_{G,(0)}^{2}=0.4443$ & $0.0456(0.00001)$ \\
$\kappa_{D}$ & $\mathcal{N}(0.5,0.25)$ & $\kappa_{D,(0)}=0.0947$ & $0.2718(0.00013)$ \\
$\sigma_{D}^{2}$ & $\mathcal{I} \mathcal{G}(3.0,1.0)$ & $\sigma_{D,(0)}^{2}=0.7930$ & $0.0762(0.00002)$ \\
$\rho$ & $\mathcal{U}(-1.0,1.0)$ & $\rho_{(0)}=0.2278$ & $0.3781(0.00017)$ \\
\hline \hline
\end{tabular}


Table 5

Estimated Parameters and Prior Distributions for the Jump-Diffusion Model

This table shows analogously to Table 4 the prior distributions, hyperparameters and starting values for all parameters of the jump diffusion model. Also, the point estimates of the parameters and their standard errors (given in brackets) are given.

\begin{tabular}{lrrr}
\hline \hline Parameter & Prior Distribution & Starting Values & Estimated Values \\
\hline$\kappa_{G}$ & $\mathcal{N}(0.5,0.25)$ & $\kappa_{G,(0)}=0.9029$ & $0.1655(0.00010)$ \\
$\sigma_{G}^{2}$ & $\mathcal{I} \mathcal{G}(3.0,1.0)$ & $\sigma_{G,(0)}^{2}=0.3679$ & $0.0134(0.00001)$ \\
$\mu_{J}^{G}$ & $\mathcal{N}(0.5,0.25)$ & $\mu_{J,(0)}^{G}=0.5342$ & $-0.0183(0.00019)$ \\
$\sigma_{J, G}^{2}$ & $\mathcal{I} \mathcal{G}(3.0,1.0)$ & $\sigma_{G,(0)}^{2}=0.3076$ & $0.1736(0.00013)$ \\
$\lambda_{G}$ & $\mathcal{B}(10,3100)$ & $\lambda_{G,(0)}=0.0018$ & $0.0944(0.00006)$ \\
$\kappa_{D}$ & $\mathcal{N}(0.5,0.25)$ & $\kappa_{D,(0)}=0.4262$ & $0.1452(0.00010)$ \\
$\sigma_{D}^{2}$ & $\mathcal{I} \mathcal{G}(3.0,1.0)$ & $\sigma_{D,(0)}^{2}=0.6219$ & $0.0144(0.00001)$ \\
$\mu_{J}^{D}$ & $\mathcal{N}(0.5,0.25)$ & $\mu_{J,(0)}^{D}=0.6100$ & $-0.0005(0.00020)$ \\
$\sigma_{J, D}^{2}$ & $\mathcal{I G}(3.0,1.0)$ & $\sigma_{D,(0)}^{2}=0.6725$ & $0.2624(0.00017)$ \\
$\lambda_{D}$ & $\mathcal{B}(10,3100)$ & $\lambda_{D,(0)}=0.0024$ & $0.1215(0.00006)$ \\
$\rho$ & $\mathcal{U}(-1.0,1.0)$ & $\rho_{(0)}=0.5017$ & $0.1934(0.00026)$ \\
\hline \hline
\end{tabular}


Table 6

Descriptive Statistics of Deviation between Monthly PTR Market and Model Prices

This table shows descriptive statistics as well as the Root Mean Squared Error (RMSE) of the spread between observed market PTR prices and their respective model prices. Model prices are calculated based on observed daily prices (Section A) and de-spike prices (Section B). A positive spread refers to higher market prices and vice versa.

\section{A. Normal Price Process}

\begin{tabular}{lrrrrrrrr} 
Model & RMSE & Mean & Median & Minimum & Maximum & Std.dev. & Skewness & Kurtosis \\
\hline Diffusion & 20.98 & -15.83 & -12.70 & -79.57 & 13.47 & 13.84 & -1.29 & 4.21 \\
Jump-Diffusion & 11.77 & -0.87 & 0.22 & -72.46 & 24.72 & 11.80 & -3.17 & 16.44
\end{tabular}

\section{B. De-Spiked Price Process}

\begin{tabular}{lrrrrrrrr} 
Model & RMSE & Mean & Median & Minimum & Maximum & Std.dev. & Skewness & Kurtosis \\
\hline Diffusion & 19.44 & -15.05 & -12.80 & -59.14 & 13.30 & 12.37 & -0.59 & 0.88 \\
Jump-Diffusion & 9.17 & 0.02 & 0.82 & -50.46 & 24.58 & 9.21 & -2.02 & 10.30 \\
& & & & & & & & \\
\hline
\end{tabular}


Figure 1

Logarithm of German and Dutch Daily Electricity Prices

This figure shows the logarithm of German and Dutch daily electricity prices between 2001 and 2008 .

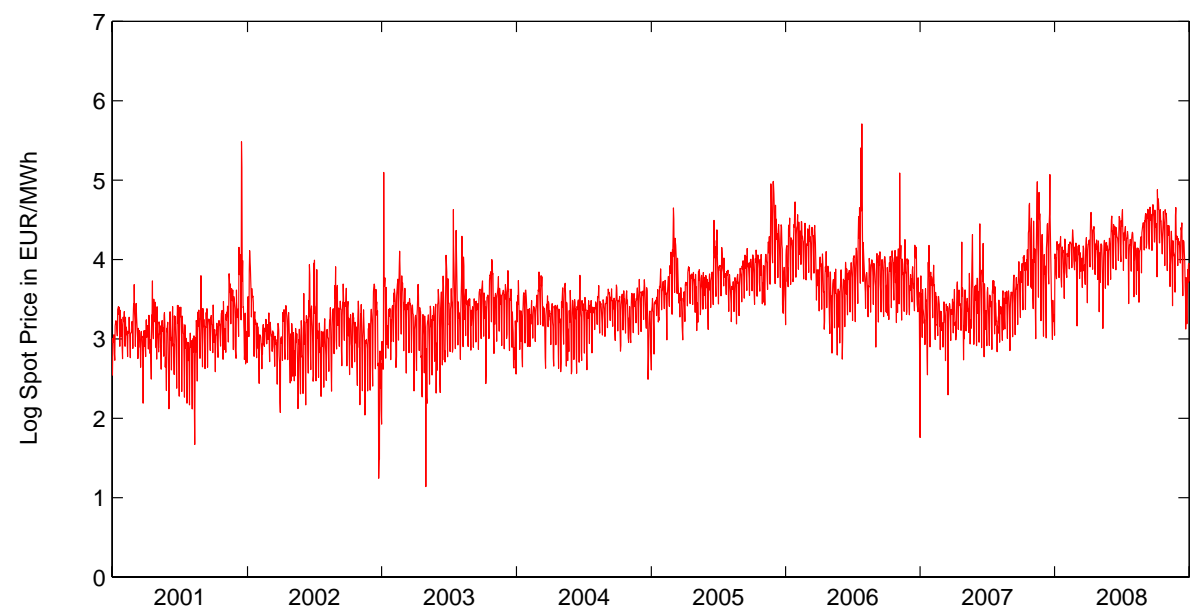

(a) Germany

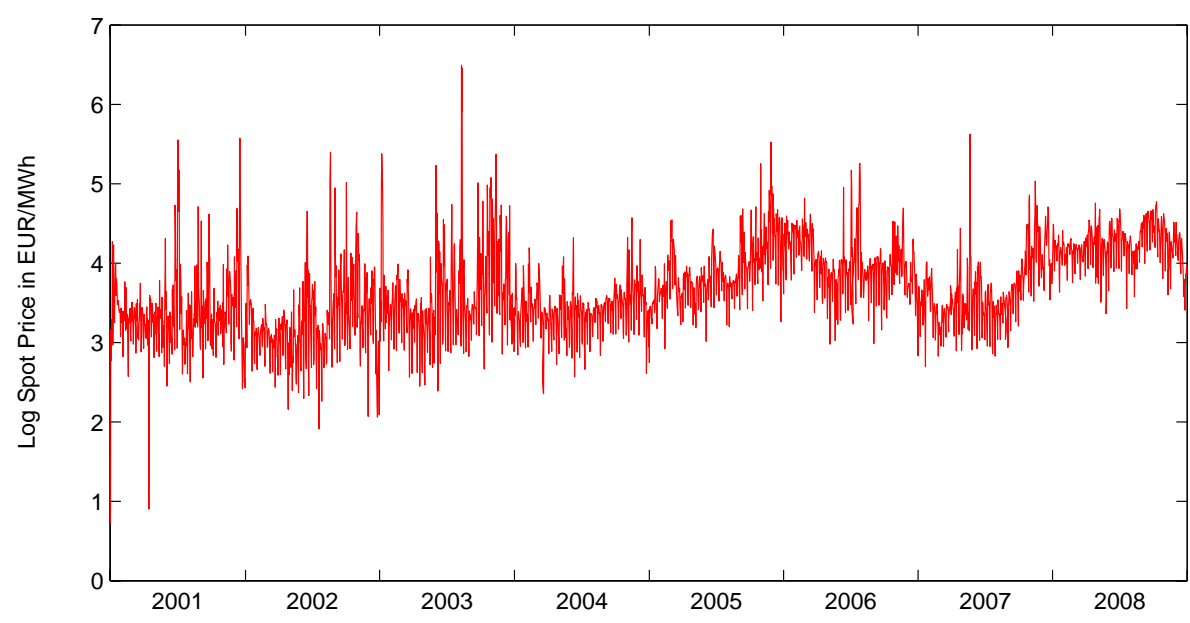

(b) Netherlands 
Figure 2

Mean PTR Model Price and Jump Intensities

This figure shows the mean PTR model price plotted against German and Dutch jump intensities. Mean model prices are calculated as the arithmetic mean of all 95 PTR option prices between February 2001 and December 2008.

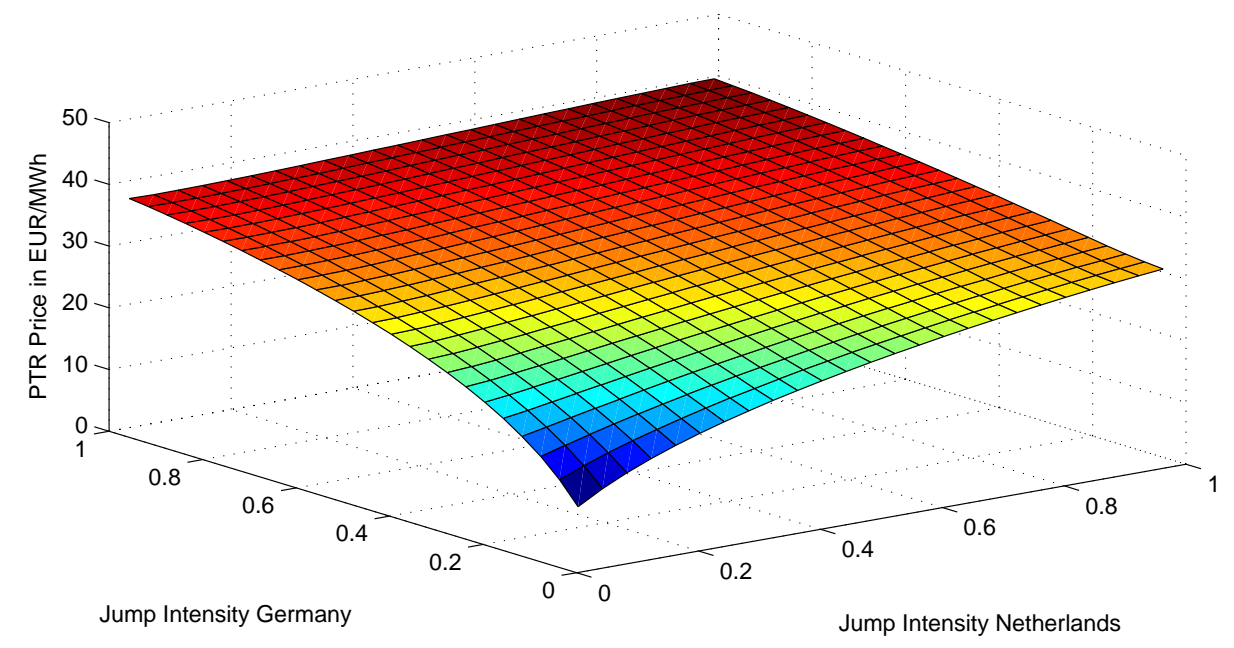


Figure 3

De-Spiked Logarithm of German and Dutch Daily Baseload Prices

This figure shows de-spiked values of the logarithm of German and Dutch daily electricity prices between 2001 and 2008. The de-spiked prices correspond the diffusion component of the underlying jump-diffusion model.

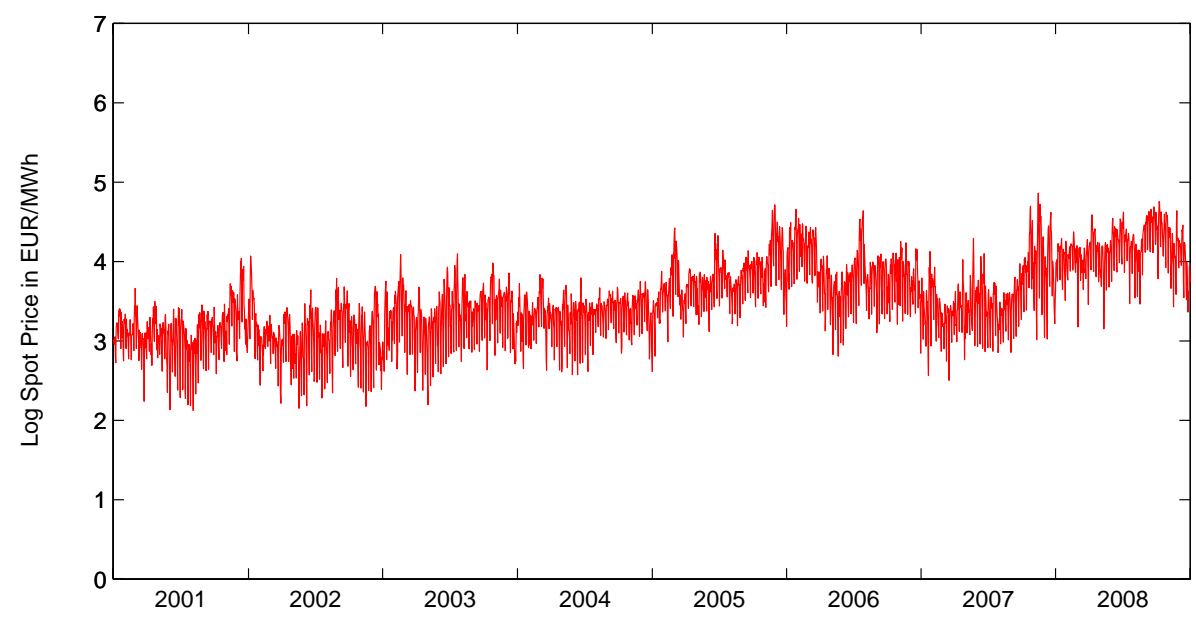

(a) Germany

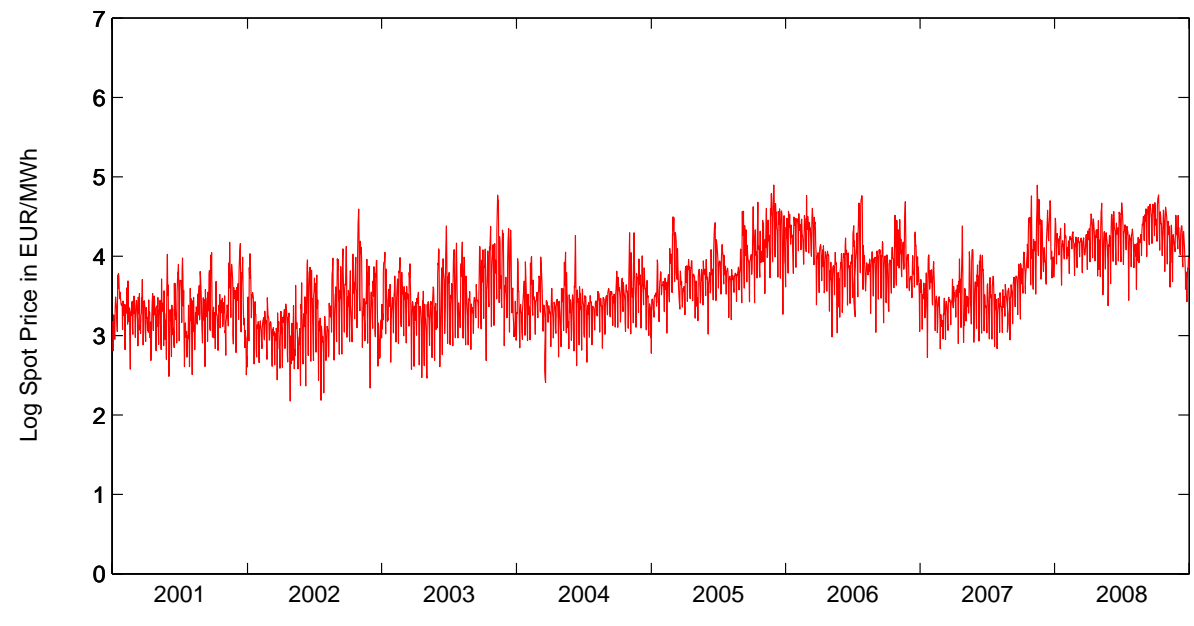

(b) Netherlands 
Figure 4

PTR Price vs. Futures Spread

This figure shows the PTR option price (dashed line) and the corresponding futures spread (solid line) for all month contracts between February 2001 and December 2008. The futures spread is calculated based on daily closing prices for physically settled futures contracts on the day of the PTR auction.

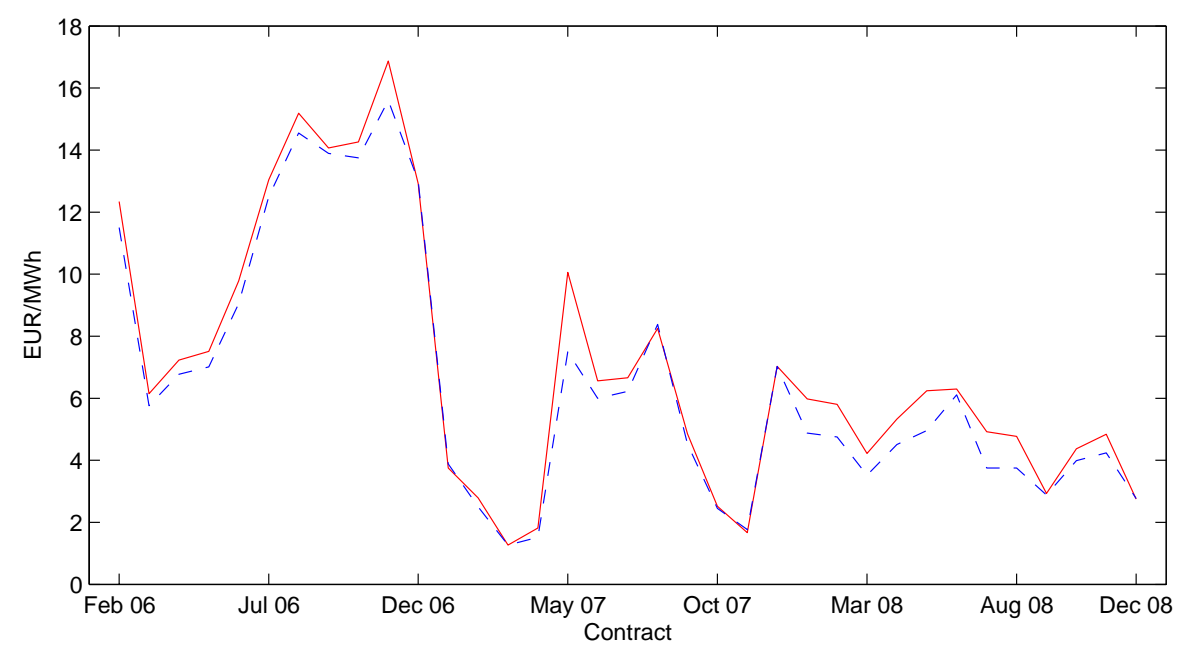

